\title{
Knöpfe, Krieg und Kapitalismus. Der Zusammenhang zwischen Armeebedarf, sozialem Militarismus und wirtschaftlicher Entwicklung ${ }^{1}$
}

\section{Einführung}

Die Finanzbürokratien der zwischen dem 16. und 18. Jahrhundert entstehenden modernen Staaten haben sich vor allem darum so nachhaltig entwickelt, weil die Herrscher auf sie angewiesen waren, um große Armeen aufzubauen, mit denen sie in Europa halbhegemoniale oder hegemoniale Stellungen erringen und außerhalb Europas schließlich Kolonialreiche errichten und militärisch sichern konnten. Der Vorgang der Herausbildung stehender Heere wurde von dem Entstehen eigenständiger Militärbürokratien begleitet, die sich u.a. bei der Beschaffung der Bekleidung von ökonomisch-zweckrationalen Prinzipien leiten ließen. Die neuentstandenen stehenden Heere boten Handwerks- und Manufakturbetrieben einen bedeutenden, ja außergewöhnlichen Markt. Große Felder für ökonomische Betätigung taten sich weit über die Produktion von Stoffen für die Bekleidung hinaus auf, wenn Menschen für die Beköstigung und die Bewaffnung der Landheere oder $z . B$. für den Schiffbau tätig werden konnten, insbesondere dann, wenn Staaten mit den entstehenden Flotten ihre Seeherrschaft sichern wollten. Für die Aufstellung, Unterhaltung und Entwicklung stehender Heere vollzog sich die Entfaltung wirtschaftlicher Aktivitäten auf ganz verschiedenen Ebenen. Der landwirtschaftliche Bereich mit Pferdezucht, Getreide- und Gemüseanbau etc., die Textilindustrie mit Montierungsteilen, Zeltstoffen, Decken usw., das' Baugewerbe mit der Errichtung von Kasernen, Festungen und Verteidigungsbollwerken, natürlich die Fahrzeugproduzenten mit ihren Erzeugnissen, aber auch das Druckgewerbe mit Büchern, Karten und sonstigen Drucksachen für den Militärbedarf und schließlich der gesamte Dienstleistungssektor etwa in Garnisonsstädten mit Gastwirtschaften bis hin zum Amüsieretablissement trugen den vielfältigen Anforderungen des Wirtschaftsfaktors Militär Rechnung. Diesen Gesamtzusammenhang schildert Werner Sombart in seiner Pionierstudie "Krieg und Kapitalismus", auf dessen im Titel angedeutete Grundintention hier durch die Wahl der eigenen Überschrift Bezug genommen wird². Hätte Sombart seine an den Verhältnissen des 18. Jahrhunderts und früherer Zeiten entwickelten Thesen auch für das 19. und 20. Jahrhundert weiterverfolgt, so wären von

1 Gekürzte und umgearbeitete Version eines Beitrages, der unter demselben Titel erschienen ist in: »Fliegt in alle Länder und empfehlt uns! - Gesellschafts- und wirtschaftsgeschichtliche Studien zur Lüdenscheider und Wuppertaler Metallknopf- und Abzeichenindustrie im 19. und 20. Jahrhundert. Festschrift für Dr. Walter Hostert zum 70. Geburtstag. Hrsg. von Eckhard Trox, Lüdenscheid 1996, S. 41-68.

2 Werner Sombart, Krieg und Kapitalismus, München, Leipzig 1913 (= Studien zur Entwicklungsgeschichte des modernen Kapitalismus, Bd 2), S. 1-14. 
ihm wahrscheinlich große Rüstungsbetriebe in den Blick genommen worden. Man assoziiert beim inneren Zusammenhang von 'Krieg und Kapitalismus auf der materiellen Ebene gewiß nicht sogleich Knöpfe und Koppelschlösser, sondern, um das konsonante Wortspiel auf die Spitze zu treiben, Krupp und Kanonen. Sombart hätte also wahrscheinlich die Schwerindustrie mit ihren großbetrieblichen Strukturen untersucht und sich wohl mit der grundsätzlichen Fragestellung befaßt, ob ein militärisch-industrieller Komplex wie etwa derjenige innerhalb des Ruhrgebietes eine kriegsfördernde Eigendynamik entwickeln könne. Der Zusammenhang zwischen 'kapitalistischer Schwerindustrie und Krieg soll in diesem Beitrag aber weitgehend unberücksichtigt bleiben.

Für neue Erkenntnisse über Struktur und Erscheinungen des Militarismus und des vielfach Besonderheiten aufweisenden deutschen Sozialmilitarismus sowie über regionale Ausprägungen und Entwicklungen des Industriekapitalismus erscheint vielmehr eine Eingrenzung der Thematik heuristisch fruchtbar. Sozialer Militarismus, dies sei vorausgeschickt, ist allgemein zu definieren als die Durchformung aller gesellschaftlichen Bereiche mit aus der militärischen Wertsphäre und aus militärischen Hierarchien stammenden Leitbildern. Diese Definition, die noch weiter zu präzisieren sein wird, soll auch für die Wahl des Untersuchungsgegenstandes herangezogen werden. Besonders sinnvoll erscheint daher die Selektion eines Spezialaspektes, der ein zivil-militärisches Doppelgesicht aufweist. Deshalb beschäftigen wir uns auf verschiedenen Ebenen mit der Uniform, vor allem der militärischen, aber auch der nicht-militärischen. Es soll gefragt werden, welche Handwerkszweige und Industrien die harten und glänzenden Teile für die Uniform produzierten. Die Uniform in ihrer vollendeten Ausformung gilt bis heute den Menschen gleichsam als das Urbild alles.Militärischen. Gemeint ist die Uniform mit ihren Knöpfen, Effekten, Orden, mit ihren Koppelschlössern und den Beschlägen für den Helm. Zweitens soll unter sozialgeschichtlicher Prämisse untersucht werden, wie dieses Urbild auf gesellschaftliche Phänomene abstrahlte. Es geht also um Aspekte des spezifisch deutschen Sozialmilitarismus, um das Problem, ob in Abzeichen, Orden und Militäreffekten, also in kulturellen Objektivationen dieses Militarismus, Besonderheiten der deutschen Gesellschaftsgeschichte erkennbar werden. Dieser Aspekt soll drittens mit der regionalhistorischen Fragestellung verknüpft werden, welche Konsequenzen die besonders im Deutschen Kaiserreich erkennbar werdende Ausweitung von Militarismus und Sozialmilitarismus für die Industrie besaß, die mit der Produktion der Kleinteile für die Uniformen, aber auch mit der Herstellung von Abzeichen für die Bedürfnisse von konservativ-militaristischen, bürgerlichen und gelegentlich auch sozialistischen Organisationen befaßt war. Es geht hier also nicht um die Bewaffnung von Armeen, nicht um Panzer oder Schlachtschiffe, es geht auch nicht um die Heraldik auf den Knöpfen und Militäreffekten, sondern um den, wie ich ihn bezeichnen möchte, dekorativen Militarismus. Hier soll nicht nach Zeichen auf den Metallteilen und deren Bedeutung gefragt werden, sondern es wird ein erneuter Versuch unternommen, den deutschen Militarismus besser zu verstehen und dessen regionalwirtschaftliche Bedeutung an einem bisher vernachlässigten Forschungsgegenstand auszuloten.

Noch im Mittelalter hatte jeder Krieger selbst für seine Bekleidung gesorgt. Doch später, in jener für den Okzident so außerordentlich bedeutsamen historischen Phase, als einheitlich ausgerüstete Heere aufgebaut wurden, trat die kollektive Bedarfsdeckung an die Stelle der Einzelversorgung. Nunmehr waren der 
Oberst des Regiments oder der Kompaniechef für die regiments- bzw. kompanieweise Beschaffung der Bekleidung verantwortlich. Allmählich griff - in Preußen insbesondere unter Friedrich Wilhelm I. - auch der Staat in das Bekleidungswesen für die Armeen ein. Er beschaffte für die Kompaniechefs oder Obersten die Tuche, welche dann zu Uniformen verarbeitet wurden ${ }^{3}$. Die Ausrüstung ihrer Garden mit häufig extrem hohen Aufwendungen besorgten die Landesfürsten zumeist selbst. Die Entwicklung hin zu einheitlichen Uniformen bei den verschiedenen Armeen in Europa war ein so differenzierter Vorgang, daß er in diesem Zusammenhang nicht dargestellt werden kann. Es ist dagegen möglich, den politisch-ökonomischen Entwicklungsprozeß, vor dessen Hintergrund sich die Entstehung und Entwicklung der Uniformierung vollzog, hier grob zu skizzieren.

Die militärische Uniform, die einen ihrer Ursprünge im Bedienstetenverhältnis hatte - weshalb sie zunächst auch noch Livree genannt wurde ${ }^{4}$ - , setzte sich in Deutschland Mitte des 18. Jahrhunderts endgültig flächendeckend durch,

3 Zum Zusammenhang vgl. den ,Klassiker، von Gustav Schmoller, Die Entstehung des preußischen Heeres von 1640-1740, in: Deutsche Rundschau, 12 (1877), S. 248-273, hier bes. S. 261 f.; ebenfalls ein >Klassiker - mit vielen Details zur Ausrüstung und Bekleidung bis hin zur minutiösen Darstellung der einzelnen Regimentsabzeichen - ist Curt Jany, Geschichte der preußischen Armee vom 15. Jahrhundert bis 1914. Bd 1: Von den Anfängen bis 1740, 2. Aufl., Osnabrück 1967, S. 763-781. Vgl. auch ders., Bd 2: Die Armee Friedrichs des Großen 1740-1763, 2. Aufl., Osnabrück 1967, S. 273-299. Bezüg. lich der militärischen Abzeichen - dazu gehören, wenn man sie als militärische Erkennungszeichen versteht, im übertragenen Sinn auch die Knöpfe - bis hin zum Beginn des 19. Jahrhunderts ist auch der 3. Band dieses Standardwerkes mit dem Zeitraum 1763-1807, ebd., S. 199-207, heranzuziehen. Vgl. auch Carl Kling, Geschichte der Bekleidung, Bewaffnung und Ausrüstung des Königlich Preußischen Heeres, 3 Bde, Weimar 1902-1912. Eine Einführung in den Zusammenhang, insbesondere Einblick in staatliche Musterbücher, über die dann die Tressen für Uniformen wiederbeschafft wurden, gewährt durch zahlreiche Abbildungen Hans Bleckwenn, Unter dem Preußen-Adler, München 1978, bes. S. 92-99. Vgl. auch ders., Die friderizianischen Uniformen 1753 bis 1786, 4 Bde, Dortmund 1984; vgl. außerdem: Der bunte Rock in Preußen. Militär- und Ziviluniformen 17. bis 20. Jahrhundert in Zeichnungen, Stichen und Photographien aus dem Bestand der Kunstbibliothek Berlin. Ausstellungskatalog, Berlin 1981. Einen Überblick über die Uniformierung der Armeen innerhalb des Deutschen Bundes unter Berücksichtigung auch der Orden, Ehrenzeichen und Knöpfe bietet der wohl bedeutendste süddeutsche Militärschriftsteller der Vormärzzeit Max Ritter von Xylander, Das Heer-Wesen der Staaten des Deutschen Bundes, Augsburg 1838. Zu Preußen vgl. bes. ebd., S. 117-153. Die Grundlagen der altpreußischen Bekleidungswirtschaft sind aufgearbeitet worden von Gisela Krause, Altpreußische Militärbekleidungswirtschaft. Materialien und Formen, Planung und Fertigung, Wirtschaft und Verwaltung, Osnabrück 1983 (= Das altpreußische Heer, T. 7, Bd 1); vgl. auch dies., Altpreußische Uniformfertigung als Vorstufe der Bekleidungsindustrie, Hamburg 1965. Das Wechselverhältnis von ziviler Männerkleidung und der Uniform wird beschrieben und diskutiert bei Eva-Maria Klein, "Weil der Knopf zum Putze des männlichen Geschlechtes gehört...«. Von der Uniform zum Arzug, in: Auf und zu. Von Knöpfen, Schnüren, Reißverschlüssen... Hrsg. von Gabriele Mentges, Stuttgart 1994, S. $45-59$.

4 Marieluise Kliegel, Des Dieners alte Kleider. Knöpfe und Livreen an Adelshöfen des 19. Jahrhunderts, Lüdenscheid 1994 (= Forschungen zur Geschichte der Stadt Lüdenscheid - Objekte und Dokumente, Bd 4). Die Autorin stellt in diesem Band u.a. zahIreiche Livreen von Bediensteten an westfälischen Adelshöfen vor. Sie wird das in der weiteren Untersuchung hier ausgeblendete Thema der Ziviluniformen in ihrer voraussichtlich 1997 erscheinenden Dissertation erheblich vertiefen. - Der Zusammenhang 
weil die stehenden Heere im Gefüge der absolutistischen Staaten immer größere Bedeutung gewannen. Preußen definierte sich durch viele Elemente seines Erscheinungsbildes, so u.a. durch die Viten seiner Könige, allmählich immer stärker auch als Militärmonarchie. Militärische Disziplin ohne Uniform galt daher bereits zu Zeiten Friedrich II. in Preußen als nahezu undenkbar. Doch neben den Erfordernissen des Militärstaates gab es vor allem ökonomische Gründe für die Uniformierung. Die Gleichförmigkeit schuf nämlich die Möglichkeit des Massenbezuges und damit der Massenherstellung. Für diejenigen Bestandteile der Uniform, für deren Erstellung spezielle handwerkliche Techniken erforderlich waren, galten diese Gesetze der Masse natürlich nur im eingeschränkten Maße. Die konfessionell-politische Zersplitterung Deutschlands im 18. und weitgehend auch im 19. Jahrhundert zwingt außerdem dazu, differenziert von den vielfältigen Geschichten der Ausrüstung einzelner deutscher Länder und dort einzelner Regimenter mit zum Teil sehr spezifischem Bedarf zu sprechen, wodurch es zu einer weiteren, der Massenproduktion abträglichen Differenzierung kam. Mit der Befriedigung des Bedarfes betraut wurde ein Spezialhandwerk, welches Knöpfe, Abzeichen und Kokarden herstellte und damit in erster Linie für die Armee arbeitete.

Zwar produzierte man Militärknöpfe - weil eben ihre Beschaffung ausschließlich dem Kompaniechef oblag - in jener Zeit häufig nur für einzelne Regimenter und somit in begrenzter Stückzahl, doch konnten auch kleinere Aufträge in der traditionalen Sphäre des salten Handwerks für längere Zeit die $>\mathrm{Nah}$ rung sichern. Wegen der zentralen Rolle der Städte Berlin und Potsdam innerhalb der preußischen Militärmonarchie siedelten sich aus naheliegenden Gründen dort zahlreiche Handwerker an, die auf dieses Sortiment, das noch um Helmbeschläge erweitert wurde, spezialisiert waren. Für die Uniformierung von 193 Soldaten wurden im 17. Jahrhundert 579 Dutzend eiserne Knöpfe besorgt; und zur Ausrüstung eines Infanteristen am Anfang des 18. Jahrhunderts gehörten in der Regel 20 Messingknöpfe. Diese Zahlen nennt Werner Sombart, doch ihre Herkunft teilt er nicht mit. Immerhin läßt sich aber über sie eine Vorstellung von Auftragsvolumina gewinnen: zu detaillierten Zahlen gelangt man, wenn man sich näher mit der opulenten uniformgeschichtlichen Literatur und mit Regimentsgeschichten befaßt. Die Aufträge zur Ausstattung nicht etwa nur einzelner Regimenter, sondern ganzer Armeen z.B. mit Messing- und Eisenknöpfen konnten, sofern ihre Vergabe nicht mehr dem einzelnen Kompaniechef oblag, mit Blick auf die Konsequenzen für die städtische oder regionale Entwicklung in einer überschaubar kleinen, traditional-handwerksmäßig geprägten Welt geradezu revolutionäre Folgewirkungen mit sich bringen, denn die ersten Vorboten der großen Industriellen Revolution des 19. Jahrhunderts machten sich bei solchen Vergaben bemerkbar. Derartige Aufträge mußten nämlich zwangsläufig Überlegungen der Produzenten nach sich ziehen, wie die angeforderten Waren schnell und effizient herzustellen waren. Șpätestens seit der Französischen Revolution 1789 mit den damals aufkommenden Volksarmeen dürfte die Entwicklung - ohne dies hier exakt nachweisen zu können - sich immer stärker in

zwischen militärischen Uniformen und den in diesem Band präsentierten Livreen, die sozialgeschichtlich in absolutistische bzw. postabsolutistische Funktionszusammenhänge bzw. Herrschaftsverhältnisse eingeordnet werden müssen, erscheint optisch evident. Vgl. ebd., S. 6 f. 
Richtung staatlicher Auftragsvergabe durch zentralörtlich angebundene Militärbürokratien vollzogen haben. Auch noch ohne industrielle Fertigungsweise bedeutete die Produktion großer Stückzahlen eines Artikels im 18. Jahrhundert im Regelfall den Aufbruch in die moderne Welt.

Parallel zu diesen Vorgängen im Bereich der Kleinteilfertigung für den Uniformbereich vollzogen sich auch in anderen Städten und Regionen vergleichbare, mit dem sich vermehrenden Armeebedarf zusammenhängende Prozesse. Aus handwerksmäßigen Strukturen heraus entwickelte sich nämlich auch andernorts allmählich eine industrielle Fertigungsweise für Produkte, die zur vollständigen Herstellung der kompletten Ausrüstung von Soldaten oder Offizieren benötigt wurden. $\mathrm{Zu}$ denken ist hier insbesondere an Städte und Landstriche, in denen Tuche, Leinen, Hüte, Stiefel, Strümpfe und Borten produziert wurden ${ }^{5}$. Zweifelsohne hatten Großaufträge von Militärverwaltungen entscheidenden Einfluß auf die Größenordnung und Entwicklung des gewerblichen Lebens in spezifischen Regionen. Städte wuchsen, Umschlagplätze wurden geschaffen, Händler und Verleger bezogen Teile der Landbevölkerung hausindustriell ein, und schließlich gestalteten sich die Beziehungen von Kaufleuten und Produzenten um. Der selbständige Handwerkerstand alten Typs konnte sich bei Massenaufträgen auf Dauer nicht bewähren und damit selbst erhalten, denn einer auf Rationalisierung und auf Maschineneinsatz bedachten, außerzünftischen Konkurrenz war er nicht gewachsen. Der deutsche Soziologe und Nationalökonom Werner Sombart resümiert: "So tritt uns auch hier [im 18. Jahrhundert, E.T.] das moderne Heer als Erzieher zum Kapitalismus entgegen ${ }^{6}$.«

Vor dem Einsetzen der Massenproduktion mit Maschinen um 1830 fertigten Handwerker Knöpfe in sehr vielfältiger Form. In der Zeit nach dem Dreißigjährigen Krieg, mit der Entwicklung vom Söldnerheer zum stehenden Heer, erfreuten sich die Knopfmacher bereits eines Massenabsatzes; nun nämlich setzte sich die Verschlußform der Knöpfung bei der Kleidung allgemein durch. Neben den in einer Zunft organisierten Knopfmachern, die, wie wir durch Walter Hosterts Forschungen wissen, eines ihrer traditionellen Zentren in Nürnberg hatten, produzierten ebenfalls Goldschmiede, Posamentierer, Schneider, Säckler, Dreher und schließlich Gürtler, Weiß- und Gelbgießer aus sehr unterschiedlichen Materialien Knöpfe. Auch in Preußen waren im 18. Jahrhundert mehrere Gewerbe und Innungen an der Knopfproduktion beteiligt, die sich teilweise gegenseitig die Rechte streitig gemacht haben dürften. Trotz unklarer Rechtslage bemühte sich der preußische Staat aber intensiv um die Ausschaltung ausländischer Konkurrenz. Im Jahre 1718, wenige Jahre nach dem Regierungsantritt Friedrich Wilhelms I., wurde in Preußen auch die Einfuhr von Metallknöpfen verboten. Wegen seines Hauptstadtcharakters erlebte Berlin im 18. Jahrhundert eine ausgesprochene Blüte der Knopfproduktion. Insbesondere die Gürtler sowie die Weißund Gelbgießer gossen und schlugen Metallknöpfe aus unedlen Metallen, die in der Regel mit Gold- oder Silberlegierungen veredelt wurden. Die veredelten

5 Sombart, Krieg (wie Anm. 2), S. 150-174. Zum Problem vgl. insbesondere auch Peter Kriedte, Hans Medick, Jürgen Schlumbohm, Industrialisierung vor der Industrialisierung. Gewerbliche Warenproduktion auf dem Land in der Formationsphase des Kapitalismus, Göttingen 1978. Die Autoren thematisieren allerdings den Zusammenhang von Hausindustrie und Militärbedarf kaum.

6 Sombart, Krieg (wie Anm. 2), S. 173. 
Knöpfe fanden primär im Uniformbereich Verwendung. Einer der wichtigsten der in Berlin ansässigen Knopfproduzenten war der Galanteriewarenhändler Pierre Fromery, der das Privileg besaß, Knöpfe für die Röcke der Offiziere des Königs anzufertigen. Ein eigentliches regionales Zentrum für die Massenproduktion von Knöpfen und Militäreffekten - abgesehen von Berlin/Potsdam gab es im 18. Jahrhundert nicht, wobei die Berliner Knopf- und Militäreffektenhersteller ähnlich wie die frühen Wiener Abzeichenproduzenten eine auf Spezialisierung setzende Handwerkstradition repräsentierten, nicht aber moderne Formen der Massenproduktion entwickelt hatten. Die »hauptsächlich in Berlin ansässigen ", hochspezialisierten "Helmfabrikanten " ließen beispielsweise noch zu Beginn des 20. Jahrhunderts ihre »Beschläge meist bei kleinen Gürtlern auf eigenen Werkzeugen « herstellen. Diese Beobachtung zeigt deutlich, daß diese Fabrikanten sich noch ganz in der handwerklichen Sphäre befanden?

Sombarts Einschätzung, daß das Heer schon im 18. Jahrhundert der Erzieher zum Kapitalismus gewesen sei, ist gewiß zutreffend. Doch in wieviel größerem Ausmaß mußten Sombarts an den Verhältnissen des 17. und 18. Jahrhunderts entwickelte Aussagen angesichts des Bevölkerungswachstums und der immensen Vergrößerung der stehenden Heere im 19. Jahrhundert und frühen 20. Jahrhundert für den teilweise innigen Zusammenhang von Militärstaatlichkeit und Militarismus auf der einen Seite und Industrieentwicklung, Veränderung der Städte und Regionen, die für den oben näher definierten dekorativen Militarismus produzieren, sowie der Vermehrung von Industriekapital insgesamt auf der anderen Seite gelten? Diese Frage zu beantworten, soll u.a. Gegenstand der folgenden Untersuchung sein. Wegen der in zeitlicher und sachlicher Hinsicht weitumspannenden Anlage des Themas sei vorab darauf hingewiesen, daß alle weiteren Erörterungen und die folgende Darstellung letztlich nur so etwas wie Prolegomena sein können, denn die Formulierung exakter Aussagen beispielsweise zu Konzentrationsbewegungen des Kapitals bei bestimmten Firmen hätte die Durchsicht von Quellenbeständen in außergewöhnlichem Umfang erforderlich gemacht, was im Rahmen dieses Aufsatzes nicht möglich war. Und allein der Versuch der Quantifizierung des Militärbedarfs eines Staates des Deutschen Bundes z.B. an Knöpfen in der Zeit zwischen 1815 und 1870 bringt quellenmäßige und methodische Probleme erheblichen Umfanges mit sich. Es wird auf diese Diffizilitäten noch einzugehen sein. Das Problem mit quantifizierenden Aussagen wird noch dadurch vergrößert, daß bereits im späten 18. Jahrhundert andere staatliche Teilbereiche ebenso wie gesellschaftliche Institutionen ihre Bediensteten

7 Zu den Berliner Helmfabrikanten vgl. den Jahresbericht der Handelskammer zu Lüdenscheid für 1904/05, Lüdenscheid 1905, S. 27. Allgemein zu den Handwerkstraditionen bei der Knopfproduktion vgl. Eva-Maria Klein, Knopfherstellung früher und heute. Das Knopfmacherhandwerk in Württemberg, in: Auf und zu (wie Anm. 3), S. 151-159, bes. S. 153 f.; zur alten deutschen Knopfmacherstadt Nürnberg vgl. Walter Hostert, Lüdenscheid und die Knöpfe. Eine kleine Kulturgeschichte des Knopfes, 2. Aufl., Lüdenscheid 1977, S. 16. Hostert kann in Nürnberg den Einsatz von Maschinen bei der Produktion von Messingknöpfen schon für das 17. Jahrhundert belegen. Eine Massenproduktion in der Fabrik, wie sie in den dreißiger Jahren des 19. Jahrhunderts in Preußen einsetzte, konnte dadurch aber offenbar noch nicht ausgelöst werden. Zur Knopfproduktion in Berlin und Potsdam vgl. die kurzen Hinweise bei Klaus-Peter Merta, Das Heerwesen in Brandenburg und Preußen von 1640 bis 1806, Brandenburg 1991, S. 46. Einen Überblick vermittelt auch Hans Schramm, Die deutsche Knopfindustrie in Geschichte, Volkswirtschaft und Weltwirtschaft, Naunhof-Leipzig 1921. 
mit Uniformen ausstatteten. Das galt für Post, Zoll, Bergbauverwaltungen u.v.a., aber auch für bürgerliche Institutionen wie etwa die Freiwilligen Feuerwehren, die Bürgergarden oder dann 1848 die Bürgerwehren ${ }^{8}$. Für das 19. Jahrhundert bis zum Ende des Kaiserreiches 1918, verstärkt aber noch während der Zeit des Nationalsozialismus, galt, daß sich qualitativ und quantitativ gesellschaftliche Entwicklungen vollzogen, die für bestimmte Industriezweige besonders interessant waren: qualitativ durch den sozialen Militarismus bzw. durch die Entwicklung gesellschaftlich prägender >Militair-Monarchien` und quantitativ durch die permanente Vergrößerung der Armeen und die Ausweitung der sozial-militarisierten Organisationen, wobei auf den Begriff des Militarismus an anderer Stelle noch näher eingegangen werden soll. Manche Städte forcierten die für sie günstige Entwicklung der Gesellschaft dadurch, daß ihre Unternehmer ein exzellentes Handwerker- und Facharbeiterpotential durch eigens hierfür geschaffene Gewerbeschulen heranzogen oder indem sie junge Kaufleute und Techniker zum Erwerb von spezifischen Kenntnissen an die Universitäten oder in fremde Städte zur >Industriespionage entsandten. Wenn diese Städte noch über kurze Wege zu den benötigten Rohstoffen verfügten, versprachen die angesprochenen, auf eine umfassende Militarisierung, aber zumindest Uniformierung der Gesellschaft hinauslaufenden Entwicklungsstränge nahezu stetes regionales Wirtschaftswachstum. Um zu spezifizierten Aussagen zu gelangen, werden wir uns zu einem späteren Zeitpunkt für eine Auswahluntersuchung entscheiden. Hier gilt es noch einmal zu betonen, daß die Phänomene, die im folgenden in den Blick genommen werden sollen, bisher viel weniger untersucht worden sind als vergleichbare Phänomene aus dem Bereich der Schwerindustrie. Schlachtschiffe, Kanonen und sonstiges schweres Geschütz fanden schon immer das Interesse von Militär-, Politik- und Sozialhistorikern, Aspekte eines gleichsam dekorativen Militarismus wurden unter wirtschafts- und sozialgeschichtlicher Fragestellung viel weniger untersucht, obwohl gewiß ist, daß für die Selbstinszenierung staatlicher und gesellschaftlicher Hierarchien, für die Darstellung von `Gesinnungen` und für die Sichtbarmachung von Mentalitäten in Deutschland namentlich im 19. und in der ersten Hälfte des 20. Jahrhunderts im erheblichen Umfang Kapital zur Verfügung stand bzw. bereitgestellt wurde. Die zum Teil außergewöhnlichen Gewinne wurden aber hier nicht von der Schwerindustrie, sondern von kleinen und mittelständisch geprägten Unternehmen erzielt.

II. Der Primat des >militärischen ‘ gegenüber dem sindustriellkapitalistischen Geist in Deutschland im Zeitalter der Industriellen Revolution und der Hochindustrialisierung

Der britische Philosoph und Sozialwissenschaftler Herbert Spencer stellte im Hinblick auf die Entwicklungsgeschichte menschlicher Kulturen den "kriegerischen « und den »industriellen Typus« gegenüber. In diesem Zusammenhang sei nach Spencer das »Grundprinzip» des »kriegerischen Typus« lediglich über das

8 Paul Martin, Der bunte Rock. In Zusammenarbeit mit Hans-Joachim Ullrich, Stuttgart 1963, S. 96-132. 
»zwangsweise Zusammenwirken « zu definieren. Da der industrielle Typus sich dagegen durch Lebendigkeit und durch seine Innovationsfreudigkeit in seinem freien Wirken entfalten könne, stehe er dem kriegerischen Typus gegenüber und repräsentiere zugleich die höhere menschliche Kulturstufe ${ }^{9}$. Werner Sombart geht es in seiner während des Ersten Weltkrieges entstandenen Schrift »Händler und Helden " um eine Synthese der beiden Kulturtypen - und dies, wie konnte es bei einem deutschen Nationalökonomen in jenen Jahren anders sein, zur Verteidigung des deutschen Typus. Einerseits versuchte sich Sombart wie viele andere deutsche Intellektuelle jener Jahre von dem als materialistisch empfundenen Liberalismus englischer Prägung abzusetzen. Andererseits meinte er seine gewünschte Synthese und damit den »deutschen Typus« nur ermitteln zu können, indem er sich vom Stereotyp eines vermeintlich übermäßig militaristischen deutschen Charakters absetzte. Hierzu distanzierte er sich zunächst von Definitionen des Militärischen, wie sie vielfach von außen an das Deutschland Kaiser Wilhelms II. - und zwar verfälschend - herangetragen würden. Man habe sich im Ausland daran gewöhnt, "bestimmte Institutionen " Deutschlands im militärisch-industriellen Komplex ${ }^{10}$, u.a. aber auch die Verfassung des Deutschen Reiches, mit dem deutschen Militarismus schlechthin zu identifizieren. Die Abschaffung dieser Institutionen - so schätzten dies viele ausländische Beobachter ein - sei die Conditio sine qua non des deutschen Aufstiegs zur Kulturhöhe etwa Englands oder der USA. Sombart sieht dagegen »alle äußere[n] Erscheinung[en] des gesellschaftlichen und staatlichen Lebens « - hier bezogen auf den deutschen Militarismus — nicht als Konsequenz eines Institutionengefüges, welches zwangsweise Zusammenwirken erzeuge, also insbesondere der militärischen Institutionen, sondern als »die notwendigen Ausstrahlungen des Geistes [...], der ein Volk beseelt ${ }^{11}$. Auch Sombart kann natürlich nicht an der Tatsache vorbeigehen, daß der Militarismus etwas "rein Äußerliches, weil Institutionelles« sei. Der Militarismus stellt sich wie selbstverständlich auch für ihn »dar in der allgemeinen Dienstpflicht; stellt sich dar in der gewaltigen Heeresmacht, gegen die jetzt ganz Europa und die halbe übrige Welt vergeblich ankämpfen; stellt sich dar in zahlreichen Kasernenhöfen und ihren mehr und minder erfreulichen 'Blüten', in militärischem Schaugepränge, in Maschinengewehren und Schnauzbärten und Strammstehen und vielen Uniformen “12. Doch, und darin bestehe die entscheidende Differenzierung, sei dieses rein Äußerliche »aus einem bestimmten Geiste erzeugt «. Dieser Geist durchdringe Deutschlands "ganzes Volkstum «, betätige »sich in tausend und abertausend Lebensäußerungen«, »auf allen $\mathrm{Ge}$ bieten« des »öffentlichen und privaten«, des »äußeren und inneren Daseins«. "Alles, was sich auf militärische Dinge " beziehe, habe in Deutschland »den Vorrang «. Sombart fährt fort:

»Wir sind ein Volk von Kriegern. Den Kriegern gebühren die höchsten Ehren im Staate. Was äußerlich in so vielen Dingen, die dem Fremden auffallen, in

9 Die Position von Herbert Spencer ist hier wiedergegeben in der Interpretation von Werner Sombart, Händler und Helden. Patriotische Besinnungen, München, Leipzig 1915, S. $82 \mathrm{f}$.

10 Nach wie vor den besten Einstieg zu diesem Begriff bieten diverse Beiträge in: Militarismus. Hrsg. von Volker Berghahn, Köln 1975.

11 Sombart, Händler (wie Anm. 9), S. 82-84. Zu Sombart vgl. Friedrich Lenger, Werner Sombart 1863-1941. Eine Biographie, München 1994.

12 Sombart, Händler (wie Anm. 9), S. 84. 
die Erscheinung tritt: unser Kaiser erscheint selbstverständlich immer in Uniform, bei feierlichen Gelegenheiten tun desgleichen auch unsere höchsten Beamten und unsere Abgeordneten, wenn sie in einem Militärverhältnis stehen; die Prinzen kommen sozusagen als Soldaten auf die Welt und gehören von Jugend auf der Armee. Alle anderen Zweige des Volkslebens dienen dem Militärinteresse. Insbesondere auch ist das Wirtschaftsleben ihm untergeordnet $\mathrm{usw}^{13}$."

Der »deutsche Typus« könne nach Sombart zwar wirtschaftlich kompetent und engagiert handeln, doch sein Handeln sei nicht materiell, sondern geistig bestimmt. Zur Beschreibung des deutschen Militarismus gehört für Sombart daher auch der Hinweis auf die gleichsam mental-habituale Verfassung der Menschen, die durch diesen geprägt würde.

"Die äußere Organisation unseres Heerwesens hat dann dahin gewirkt, daß die geistige und körperliche Disziplin in alle Volkskreise eingedrungen ist und somit heute auch im realen Sinne einen festen Bestandteil des deutschen Volksgeistes bildet. Nicht nur im Bereiche der Armee: auf allen Gebieten unseres öffentlichen Lebens und im privaten Leben jedes einzelnen Deutschen hat sich dieser Geist der Zucht und Ordnung eingebürgert. Ob es sich um die Volksschule oder die Universitäten, um die Arbeitervereine oder die Reichsbank, um die Eisenbahnen oder die Wissenschaft handelt: es ist immer derselbe Geist, es ist immer der deutsche 'Militarismus', der sie beseelt, vor dem der Fremde wie vor einem Wunder steht. Denn aus diesem Geiste werden die Riesenwerke der Organisation geschaffen, die in diesem Kriege wiederum die Welt in Erstaunen versetzt hat.«

Was Sombart hier 1915 beschreibt, war der in Deutschland sich zunächst im Vormärz primär in Preußen ausprägende, durch vielfache Quellenzeugnisse belegbare >militairische` oder ’kriegerische Geist`, der sich eben gerade in Preußen zu einer politisch-milieuprägenden Größe sui generis ausformte. Dieser Militarismus ist geistesgeschichtlich in seiner kontinentaleuropäischen Variante nichts anderes als eine sich auf das zivile Leben auswirkende, affirmativ verstärkende Reaktion der Menschen auf die Herausbildung stehender Heere in sich alsbald häufig als Militärmonarchien definierenden Staaten, in der preußisch-deutschen und damit wirkungsmächtigsten Variante darüber hinaus eine spezifische Spielart konservativen Denkens und somit auch eine abwehrende Reaktion auf die emanzipatorischen Potenzen der Aufklärung. Diese teils aus der Auseinandersetzung mit Sombarts Thesen, teils aus eigenen Forschungen abgeleitete Definition $^{14}$ soll durch ein Abgrenzungsverfahren auf ihre Plausibilität überprüft wer-

4 Ebd., S. 86. Vgl. hierzu das Kapitel »Der >militairische Geist^. Spielarten konservativen Denkens in einer vom Militärischen geprägten Gesellschaft « bei Eckhard Trox, Militärischer Konservativismus. Kriegervereine und »Militärpartei « in Preußen zwischen 1815 und 1848/49, Stuttgart 1990, S. 93-98. Vgl. auch ders., Kriegerfeste, militärische Männerbünde und politische Offiziere. Aspekte preußischer Militärgeschichte in der ersten Hälfte des 19. Jahrhunderts als Geschichte konservativer Modernisierung, in: MGM, 51 (1992), S. 23-46. Daß es sich beim 'militärischen Geist um eine preußische Besonderheit des konservativen Denkens handelt, verdeutlicht via negationis am süddeutschen Beispiel ders., Heinrich Elsner. Vom Jakobinismus zum Konservativismus. Ein Beitrag zur Entstehungsgeschichte der konservativen Partei in Württemberg, in: Zeitschrift für Württembergische Landesgeschichte, 52 (1993), S. 303-335. Die theoretischen Grundlagen der Begrifflichkeit werden diskutiert bei Samuel P. Huntington, The 
den. Hierzu wenden wir uns sozialdemokratischen Organisationen in PreußenDeutschland zu. Wenn sich deren zuweilen uniformierte Mitglieder militärisch diszipliniert verhielten, ist es nicht sinnvoll, sie nach dieser Definition als sozial militarisiert zu charakterisieren, sofern sich diese Organisationen auf emanzipatorisch-aufklärerische Positionen beriefen - und das war zweifellos der Regelfall. Trugen solche Organisationen Abzeichen und andere Erkennungszeichen, waren sie nicht von einem militärischen Geist geprägt, sondern lediglich in den Formen ihrer Selbstdarstellung von bürgerlichen Organisationen beeinflußt. Die spezifische Struktur von Vereinen und Parteien, die ja immer eine Trennung in Vorstand und 'Restverein', die Bestimmung von Ordnern, die Bildung von Komitees und Ausschüssen mit sich brachte, unterwanderte - der Verein war ja gewissermaßen eine ১bürgerliche Erfindung - das sozialistischer Theorie inhärente kollektivistische Miteinander. Jenes war mit dem ersten Abzeichen für einen sozialdemokratischen Saalordner oder gar einer Uniform gleichsam seiner Unschuld beraubt.

Die gegenseitige Verschränkung und gewissermaßen auch Inspirierung von industrieller Entwicklung und der sich in Deutschland ab 1870/71 zunächst ständig dynamisierenden Wirkmächtigkeit des deutschen Militarismus auch und gerade in seinen zivilen äußeren Erscheinungen läßt sich in denjenigen Städten, deren Industrie der Produktion der Symbole und Abzeichen des Militarismus sich vorrangig widmete, besonders eindrucksvoll nachweisen. Als Beispiel wähle ich hierfür die Stadt Lüdenscheid. Sie war cum grano salis für anfordernde Armeen und zivile Organisationen eine Nahtstelle, für derartige Bestellungen in der bergisch-märkischen Industrieregion der Mittelpunkt, mit Blick auf die Produktvielfalt so etwas wie ein Zentralort und zudem ein kreatives Zentrum des äußeren Erscheinungsbildes des deutschen Militarismus, auch und gerade in seiner zivilen Version. Dies gilt insbesondere für die sharten` Teile der Uniform bzw. für die Orden und Ehrenzeichen am >Festrockı des Bürgers. Lüdenscheid wurde häufig als 'Knopfstadt t bezeichnet - in sehr starkem Maße war sie das für den Modebereich. Und auch andere Produkte wurden dort hergestellt - es sei hier lediglich an die Milliarden von Schnallen und Spangen erinnert. Eine solche Charakterisierung ist an dieser Stelle besonders wichtig, denn es soll der Eindruck vermieden werden, als sei nur für den dekorativen Militarismus produziert worden. Die Stadt galt zu Recht als einer der herausragenden Orte in der Welt, in denen durch die hohe Kunst der Graveure die Embleme, Symbole und heraldischen Motive in die von allen nur erdenklichen gesellschaftlichen Organisationen, Modebewegungen und sonstigen Abnehmern gewünschte Form gebracht wurden. Und durch die Fähigkeit der Industriebetriebe zu einer immer wieder mit Innovationen aufwartenden, diversifizierten Kleinteilproduktion - zumeist aus Metall, durch langjährig gewachsene Beziehungen $\mathrm{zu}$ in- und ausländischen Beschaffungsämtern und vielen Vereinen, Verbänden und Parteien war Lüdenscheid viele Jahrzehnte in Deutschland der Inbegriff ei-

Soldier and the State. The Theory and Politics of Civil-Military Relations, Cambridge, Mass. 1957. Zum Definitionsansatz, der sich wegen der vorhandenen Nähe an der klassischen Definition des Konservativismus anlehnt, vgl. auch Karl Mannheim, Das konservative Denken. Soziologische Beiträge zum Werden des politisch-historischen Denkens in Deutschland, in: Archiv für Sozialwissenschaft und Sozialpolitik, 57 (1927), T. 1: S. 68-142; T. 2: S. 470-540. 
ner blühenden Industriestad $t^{15}$. Aber - und das ist hier zu betonen - seit dem Fundamentalwandel des politisch-historischen Selbstverständnisses der Deutschen nach 1870/71 und der Militarisierung auch anderer Staaten verlieh Lüdenscheid vielen der sich zusehends mehr militarisierenden und uniformierenden Gesellschaften das spezifische Aussehen. Die Gesellschaft des Deutschen Kaiserreichs, in der vielfach der hohe Offizier mehr galt als der Minister, ließ sich in Lüdenscheid auch für ihre zivile Sphäre Ab- bzw. Ehrenzeichen kreieren, mit der der Nimbus der militärischen Hierarchie optisch gleichsam ins Alltägliche verlängert werden konnte. Unzählige gesellschaftliche Bereiche wurden in PreußenDeutschland ja insbesondere während des Deutschen Kaiserreichs mit aus der militärischen Wertsphäre und aus militärischen Hierarchien stammenden Leitbildern durchformt. Daß Lüdenscheid dabei schon frühzeitig als Lieferant für die vermeintlich oder tatsächlich übertriebenen Wünsche des Militärstaates fungierte, bemerkte ein aufmerksamer Zeitgenosse aus dem Blickwinkel der ssocialen Democratie، schon im Vormärz. Der aus Lüdenscheid stammende Karl Grün, Übersetzer und Propagandist der Ideen Proudhons und Gegenspieler von Marx, monierte beispielsweise, daß die Uniformen der königlichen Garde und der »Lakaien« Friedrich Wilhelm IV. mit vergoldeten Knöpfen ausgestattet wurden, von denen er wußte, daß viele aus seiner Geburtsstadt kamen. Dies bezeichnete Grün angesichts der katastrophalen sozialen Verhältnisse bei der pauperisierten Bevölkerung des Vormärz als einen unvertretbaren Luxus ${ }^{16}$. Wie verbreitet der Standpunkt Karl Grüns zu jener Zeit war, steht allerdings dahin.

15 Zur jüngeren Lüdenscheider Stadtgeschichte vgl. insbesondere Günther Deitenbeck, Geschichte der Stadt Lüdenscheid 1813-1914, Lüdenscheid 1985; Walter Hostert, Die Entwicklung der Lüdenscheider Industrie vornehmlich im 19. Jahrhundert. Vom Ende des 18. Jahrhunderts bis zum Ausbruch des Ersten Weltkrieges, Lüdenscheid 1960; Wolfgang Herbig, Wirtschaft und Bevölkerung der Stadt Lüdenscheid im 19. Jahrhundert, Dortmund 1977; zu den Biographien zahlreicher Lüdenscheider (Knopf-)Fabrikanten sowie ihrer Familien vgl. Lüdenscheider Porträts I. Zur Sozialgeschichte der Porträtkunst in einer preußischen Industriestadt vom Ausgang des 18. Jahrhunderts bis nach dem Ersten Weltkrieg. Bearb. von Eckhard Trox, Lüdenscheid 1994 (= Forschungen zur Geschichte der Stadt Lüdenscheid - Objekte und Dokumente, Bd 3); zur städtischen Organisationsgeschichte vgl. Fahne und Verein. Lüdenscheider Vereine und ihre Fahnen von den Anfängen bis 1933. Bearb. von Eckhard Trox, Lüdenscheid 1993 (= Forschungen zur Geschichte der Stadt Lüdenscheid - Objekte und Dokumente, Bd 2). Zur Weimarer Zeit und insbesondere zur Geschichte des Dritten Reiches vgl. Hans-Günter Schmidt, "Es war die uns von Gott gegebene Obrigkeit«. Studien und Materialien zur Zeit des Nationalsozialismus in Lüdenscheid, Pfaffenweiler 1993.

16 Zu Karl Grün und seinem ökonomisch motivierten Antimilitarismus vgl. Karl Grün, Criminal-Procedur gegen Dr. Grün und 22 Genossen wegen Hochverraths resp. Plünderung des Zeughauses zu Prüm. Begonnen am 7. Januar 1850, S. 72-75; allgemein zu Grün mit umfangreicher Darstellung des völlig zu Unrecht in den Hintergrund gedrängten ssocialen Democraten a aus Lüdenscheid vgl. Eckhard Trox, Karl Grün (1817-1887). Eine Biographie, Lüdenscheid 1993 (= Forschungen zur Geschichte der Stadt Lüdenscheid - Objekte und Dokumente, Bd 1), bes. S. 66 f. 


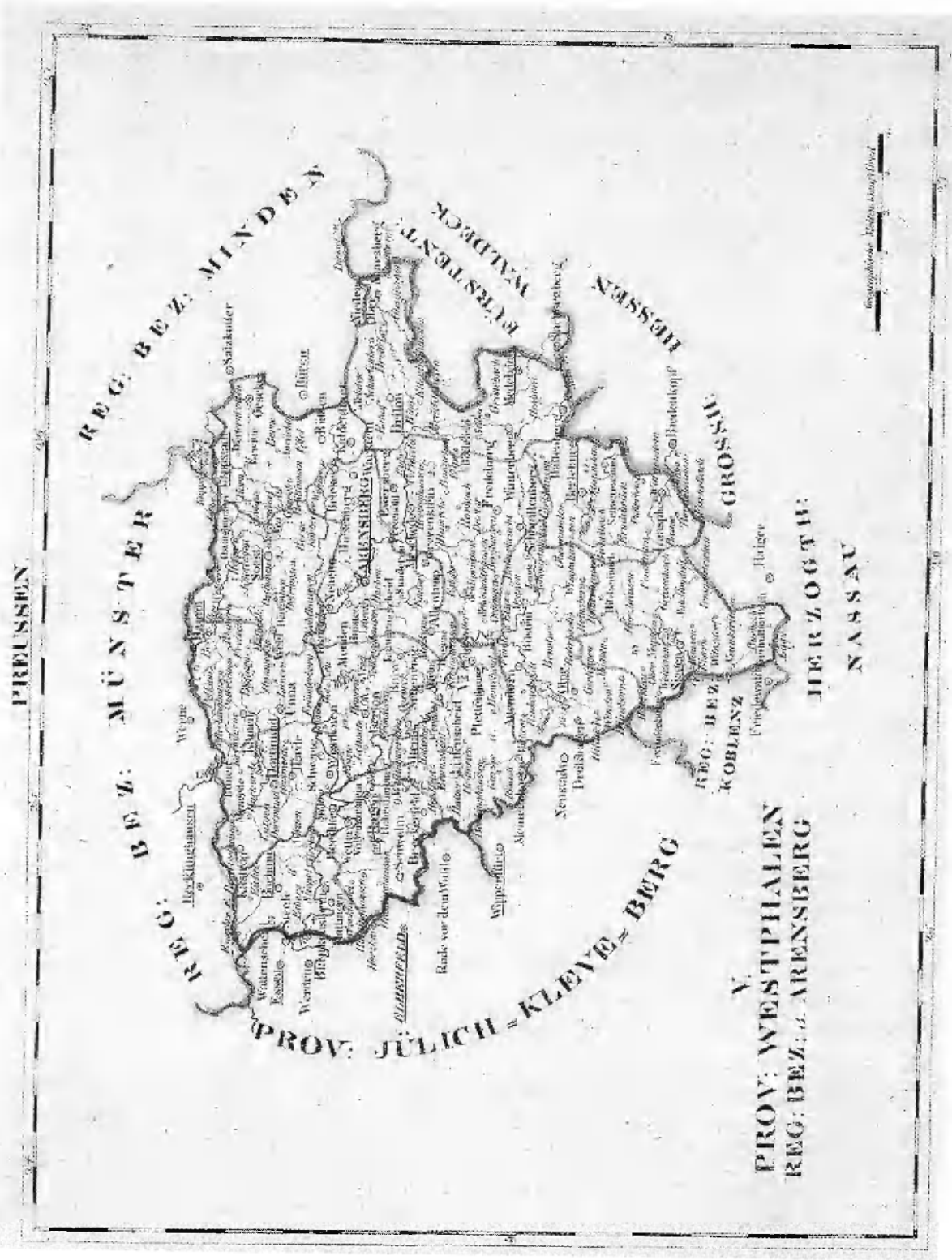




\section{Lüdenscheid - deutscher Zentralort der Kleinteilindustrie für den dekorativen Militarismus}

Eine Produktion von Knöpfen, Kokarden, Abzeichen und Orden für den Heeresbedarf in eigens dafür eingerichteten Heereswerkstätten hat es - soweit ich die Literatur überblicke ${ }^{17}$ - nicht gegeben. Viele Produkte wurden, wie oben geschildert, in Berlin/Potsdam in der traditionellen Sphäre gleichsam im Dunstkreis der königlichen Garde mit wenig Rücksicht auf die entstehenden Kosten von Handwerkern gefertigt. Doch Preußen, schon lange sich immer stärker als Militärstaat definierend und insofern an Uniformzubehör interessiert, ließ seit den 80er Jahren des 18. Jahrhunderts durch seinen Wirtschaftsbeauftragten für die Grafschaft Mark, dem sogenannten Fabrikenkommissar, die Entwicklung in Lüdenscheid beobachten, die von Innovationen bei der Produktion von Knöpfen und Schnallen gekennzeichnet war. Die Kriegs- und Domänenkammer in Hamm erließ daher bereits am 15. Januar 1795 eine »Ordnung für die Schnallen-, Knopfund Bügelfabrik« der Stadt Lüdenscheid. Die einzelnen Bestimmungen und ihre Wirkungen ${ }^{18}$ können hier unberücksichtigt bleiben, wichtig war, daß der Staat nach zahlreichen Unternehmensgründungen in Lüdenscheid seinen dirigistischen Aufgaben nachkam, Regelungen herbeiführte, als Militärstaat deutliches Interesse an Knöpfen etc. bekundete und dadurch nolens volens Lüdenscheid zur ersten Knopfstadt der Monarchie erhob, wobei der alte Produktionsschwerpunkt Berlin/Potsdam staatlicherseits noch einigen Schutz erhielt, ja der >Export von Lüdenscheider Waren in der Zeit vor der französischen Herrschaft noch an der Elbe seine Grenze fand. Überhaupt hatte Preußen den märkischen Wirtschaftsraum erst'nach dem Siebenjährigen Krieg stärker gefördert, zuvor weitgehend unbeachtet gelassen. In den ersten ca. 30 Jahren des 19. Jahrhunderts vollzog die Knopfproduktion keine stetige Entwicklung, denn einerseits waren die Kriegskontributionen Napoleons im Gebiet der vormaligen Grafschaft Mark äußerst umfangreich. Andererseits scheinen die Lüdenscheider Knopffabrikanten - dies legen mündliche Überlieferungen nahe - an Napoleons Kriegszügen und dem gesteigerten Uniformausrüstungsbedarf der sich im Kriege befindlichen europäischen Staaten zwischen ca. 1800 und 1815 vorzüglich verdient zu haben. Die Anfangsjahre nach der Erneuerung der preußischen Herrschaft im südlichen Westfalen nach 1815 stürzten die Produzenten in Lüdenscheid noch einmal in eine tiefe Krise, zumal die vitale englische Knopfindustrie nach Aufhebung der Kontinentalsperre große Warenmengen in einer Art Schleuderkonkurrenz auf den europäischen Festlandsmarkt warf. Gleichwohl: unstetig, aber doch erkennbar machte die Industrie Fortschritte: Nachhaltig wirkte sich aus, daß Lüdenscheider Fabrikanten ihre Produktionsmethoden ständig verbesserten. Ganz zeittypisch arbeitete man auch mit einer frühen Form der Industriespionage ${ }^{19}$;

17 Vgl. dazu August Genth, Die preußischen Heereswerkstätten, ihre Entwicklung, allgemeine volkswirtschaftliche Bedeutung und ihr Übergang in privatwirtschaftliche Betriebe, Diss. phil. Berlin 1926; Adalbert Mila, Geschichte der Bekleidung und Ausrüstung der Königlich Preußischen Armee 1808-1878, Berlin 1878; Hans Helfritz, Geschichte der Preußischen Heeresverwaltung, Berlin 1938. Alle Autoren bieten weiterführende Literatur.

18 Hostert, Entwicklung (wie Anm. 15), S. 43-45.

19 Vgl. dazu z.B. Wolfhard Weber, Friedrich Harkort und der Technologietransfer zwi- 
Johann Peter Sandhövel beispielsweise schickte auf seine Kosten zwei junge Techniker nach Paris, die in den dortigen Fabriken neue Produktionsmethoden erkunden sollten. 1833/34 kam über Barmen erneut eine Innovation der Methode der Knopfproduktion nach Lüdenscheid ${ }^{20}$. Immer häufiger setzten die Fabrikanten auch Maschinen ein. Nun wuchs die Stadt, Bevölkerung wanderte $\mathbf{z u}^{21}$, die Fabrikanten zogen ihre zuvor z.T. noch in Heimarbeit arbeitenden Mitarbeiter in Werkstätten zu Belegschaften zusammen. Kurz: In Lüdenscheid vollzog sich der erste Abschnitt der Take-off-Phase der Industriellen Revolution relativ frühzeitig, auch wenn die erste Dampfmaschine zur Produktion der für die Knopfherstellung benötigten Maschinen erst 1845 und im Vergleich mit anderen Städten relativ spät aufgestellt wurde.

IV. Der Aufstieg - die Lüdenscheider Industrie und ihre Abhängigkeit von gesellschaftlichen Entwicklungen und politischen Denkströmungen

Im Rahmen der weiteren Darstellung soll auf einige Aspekte der wirtschaftlichen Entwicklung in der Stadt Lüdenscheid eingegangen werden. Dabei steht zunächst die Zeit von den Reichseinigungskriegen bis zum Ende des Ersten Weltkrieges im Mittelpunkt der Darstellung. Dem Zusammenhang des wirtschaftlichen Entwicklungsprozesses mit spezifischen gesellschaftlich-politischen Rahmenbedingungen gilt dabei gesteigertes Interesse. Es wird zu verfolgen sein, wie die Wachstumstrends im Bereich des Militärbedarfs und die Ausweitung der sächlichen Bedürfnisse bei den für die Gesellschaft des Deutschen Kaiserreichs typischen militaristischen Organisationen auf der einen Seite mit der erstaunlichen Entwicklung der Lüdenscheider Industrie und der sich im Rahmen des kapitalistischen Systems vollziehenden Prozesse weg von primär Mode - hin zu primär Militärartikeln auf der anderen Seite korrelierten: der Zusammenhang zwischen 'Krieg, Knöpfen und Kapitalismus ‘ dürfte dann evident werden.

Außer auf modebedingte Schwankungen hatte die "Knopfstadt $\star^{22}$ mit der spezifischen Struktur der in ihr gefertigten Produkte vor allem auf neue Strömun-

schen England und Deutschland 1780-1830, in: Bürgerlichkeit zwischen gewerblicher und industrieller Wirtschaft. Beiträge des wissenschaftlichen Kolloquiums anläßlich des 200. Geburtstages von Friedrich Harkort vom 25. bis 27. Februar 1993. Hrsg. von Wolfgang Köllmann, Wilfried Reininghaus und Karl Teppe, Dortmund 1994, S. 129-148.

20 Hostert, Entwicklung (wie Anm. 15); vgl. auch Wilhelm Romland, Entwicklung und Lage der Metallkurzwarenindustrie in den Städten Lüdenscheid und Iserlohn, Diss. jur. Freiburg 1903, S. 12 f.

21 Herbig, Wirtschaft (wie Anm. 15), S. 74-101.

22 Hostert, Entwicklung (wie Anm. 15), S. 83. Hans-Ulrich Wehler, Deutsche Gesellschaftsgeschichte. Bd 2: Von der Reformära bis zur industriellen und politischen "Deutschen Doppelrevolution« 1815-1845/49, 2. Aufl., München 1989, ordnet die Lüdenscheider Produkte im Kapitel »Die Frühindustrialisierung: Der beschwerliche Aufstieg des deutschen Industriekapitalismus « in die Wirtschaftsgeschichte Deutschlands während der ersten Hälfte des 19. Jahrhunderts ein (S. 78) und bezieht sich dabei expressis verbis auf Hosterts Dissertation (S. 802, Anm. 25). 
gen in der deutschen Gesellschaft, auf Veränderungen der außenwirtschaftlicher Rahmenbedingungen - insbesondere bei der Zollpolitik - , auf grundstürzend Neuordnungen im politischen System von Staaten flexibel zu reagieren. Als typi. sche Beispiele für den letzten Fall seien zwei Jahreszahlen genannt, an denen hier im Vorgriff - prägnant veranschaulicht werden kann, was politische Systemwechsel oder Revolutionen bewirkten. Gemeint sind die Jahre 1848 und 1933. Als im Frühjahr 1848 in den Staaten des Deutschen Bundes polyzentrisch, mit sehr disparaten politischen Zielvorstellungen verbunden, eine Revolution ausbrach, waren die Farben Schwarz-Rot-Gold gleichsam das solidaritätsstiftende Ferment, auf deren Symbolkraft man sich sowohl großdeutsch als auch kleindeutsch und unter Einschluß eines breiten gesellschaftlich-politischen Spektrums einigen konnte. Daher produzierte die Lüdenscheider Firma P.C. Turck in der Zeil "vom Frühjahr bis Herbst die Artikel Knöpfe, Kokarden und Abzeichen mit den 3 deutschen Farben oder diese auch verbunden mit den betreffenden 2 Landesfarben der deutschen Einzelstaaten «. Obwohl die Revolution, wie viele Zeitgenossen übereinstimmend berichteten, zum Teil fatale Folgen für >Handel und Wandel hatte und vielerorts die Arbeiter revolutionsbedingt entlassen wurden, bestand nach diesen Lüdenscheider Artikeln "eine so bedeutende Nachfrage«, "daß damit der größte Thẹl der Arbeiter beschäftigt werden konnte « ${ }^{23}$. Unter ökonomischen Gesichtspunkten gewiß einschneidender und für die Lüdenscheider Industri€ noch vorteilhafter war das Jahr 1933, denn im Jahr der snationalen Revolution sollten bzw. mußten die in der NSDAP oder in parteinahen Vereinen, Verbänden und Körperschaften organisierten >Volksgenossen ‘ häufig vollständig neu mit Hakenkreuzemblemen ausgerüstet werden. Massenhafte Neueinstellungen, Ausweitung der Produktionsstätten und vorher nicht übliche Langzeitschichten bis Mitternacht waren die Konsequenz, wenngleich hier natürlich auch die positiven Auswirkungen der Überwindung der Weltwirtschaftskrise sichtbar wurden ${ }^{24}$.

Doch betrachten wir zunächst einmal die Zeit um die Jahrhundertwende. Manche größere lokale Firma exportierte um 1900 Knöpfe und Schnallen nach Spanien, Österreich, Südamerika, Indien, England, Schweden, Dänemark, Nordamerika, Belgien, Rußland, Norwegen, Brasilien, Holland, Frankreich, der Schweiz, Kanada und der Türkei ${ }^{25}$. Spätestens jetzt dürfte sich in der Lüdenscheider Bevölkerung die amüsant-ironisierende Legende ausgebildet haben, "daß der erste Mensch, den Kolumbus in Amerika angetroffen habe, ein Lüden-

Zur Einhundertjährigen Jubelfeier des Fabrikgeschäftes von P.C. Turck Wwe. am 7. November 1891, Lüdenscheid 1891, S. 12.

24 Schmidt, Obrigkeit (wie Anm. 15), S. 96, präsentiert die Zahlen der Neueinstellungen bei den in Rede stehenden Firmen. Der bereits in den zwanziger Jahren sich andeutende, durch die Entstehung einer UnzahI neuer (vor-)politischer Organisationen erkennbar werdende, aber durch Hitlers Machtübernahme massiv verstärkte Boom dürfte, sofern Schmidts Zahlen zutreffen, außergewöhnlich gewesen sein. Die Firmen Assmann \& Söhne, W. Deumer, Paulmann \& Crone, Steinhauer \& Lück, Cramer \& Co., C.H.D. Dicke, Berg \& Nolte, Schröder \& Co sowie Baumeister \& Co. stellten in den ersten Monaten des Jahres 1933 allein 678 neue Mitarbeiter ein. Den stärksten Zuwachs erfuhr die Firma F.W. Assmann \& Söhne mit allein 250 Neueinstellungen. Bei einer Einwohnerzahl von rund 35000 im Jahr 1933 ist der Gesamtzuwachs bemerkenswert. Den Hinweis auf die Schichten bis Mitternacht verdanke ich dem freundlichen Hinweis eines Lüdenscheider Unternehmers, der Zeitzeuge war.

25 Unverzeichnetes Archiv der Firma P.C. Turck GmbH \& Co KG, Metallwarenfabrik; hier: Bilanzen. 
scheider Knopffabrikant gewesen sei « ${ }^{26}$. Die sich darin ausdrückende Tatsache, $\mathrm{da} ß$ es der lokalen Industrie gelungen war, die produzierten Waren weltumspannend zu exportieren, beschrieb aber nur einen Entwicklungstrend.

In den letzten drei Jahrzehnten des 19. Jahrhunderts nämlich vollzogen sich in der Gesellschaft des Deutschen Kaiserreichs Veränderungen, die in Lüdenscheid zu einer merklichen Umstrukturierung im Produktionsprofil etablierter Firmen, dazu auch zu einer erkennbaren Zahl von Neugründungen, führten. Die Begründung des Deutschen Kaiserreichs 1870/71 veränderte Deutschlands Stellung in der Welt, was bis in kleinste Lebensvollzüge zurückwirkte. Der Militarismus, »demzufolge das Militär nicht nur an die Spitze der Prestigeskala rückte, sondern mit seinen Wert- und Ehrvorstellungen, seinen Denk- und Verhaltensweisen die ganze Gesellschaft durchdrang “, erlebte 1870/71 nach seinen erfolgreich überstandenen »Kraftproben « - nämlich der Niederschlagung der Revolution von 1848/49 unter zumindest in Preußen maßgeblicher Beteiligung konservativ-militärischer Krieger- und Veteranenverein ${ }^{27}$ und der weitgehenden verfassungsmäßigen Exemption der Armee durch den preußischen Verfassungskonflikt 1862 - einen Aufschwung, der sozusagen den "kleinbürgerlichen Gesinnungsmilitarismus« an die Spitze der gesellschaftlich gewünschten Wertsysteme brachte ${ }^{28}$. Die veränderte Rolle Preußen-Deutschlands im Konzert der Mächte nach 1866 und 1870/71, die prädominante Position eines Militärstaates in Mitteleuropa und die dort gepflegten historisch-militaristischen Traditionen - der Reichskanzler Bethmann Hollweg nahm Jahre später als Major an der königlichen Tafel unterhalb der Obersten und Generäle Platz, die Kriegervereine trugen mit der Gestaltung ihrer Fahnen und Abzeichen das Symbolrepertoire der Armee bis in den letzten Winkel der Monarchie --, dies alles brachte, wie Gerhard Ritter kurz nach dem Zweiten Weltkrieg feststellte, einen gesamteuropäischen Militarisierungsprozeß hervor bzw. verstärkte ihn, da nun viele andere Staaten gleichsam in ein Konkurrenz- und Nachahmungsverhältnis zu dem deutschen $>$ Siegerstaat traten $^{29}$. Doch neben und ergänzend zu diesem Militarismus gab es ein insgesamt geändertes Selbstbewußtsein in der deutschen Gesellschaft und ihren Organisationen nach 1870/71, das sich auch in der Selbstwahrnehmung der Menschen in den von ihnen vielfach mit großer Leidenschaft gepflegten Vereinen aller nur erdenklichen Richtungen äußerte. Viele sahen sich als Mitvollstrecker der seit dem Vormärz in der Nationalbewegung angestrebten Einigung Deutschlands. Stellvertretend für viele andere goß der Historiker Heinrich Sybel am 27. Januar 1871 in einem Brief an Hermann Baumgarten dieses neue Gefühl in eine literarisch hochkarätige, oft zitierte Wendung um: »Wodurch hat man die Gnade Gottes verdient, so große und mächtige Dinge erleben zu dürfen? Und wie wird man nachher leben? Was zwanzig Jahre der Inhalt alles Wünschens und Strebens gewesen, das ist nun in so herrlicher Weise erfüllt ${ }^{30} !$ « Nach einem ersten Anlauf 1848/49 entstand im protestantisch domi-

26 Hostert, Lüdenscheid (wie Anm. 7), S. 37.

27 Trox, Konservativismus (wie Anm. 14), und ders., Kriegerfeste (wie Anm. 14).

28 So bereits Hans-Ulrich Wehler, Das Deutsche Kaiserreich 1871-1918, 3. Aufl., Göttingen 1977, S. 158-165.

29 Gerhard Ritter, Staatskunst und Kriegshandwerk. Das Problem des Militarismus in Deutschland, Bd 1, München 1954, S. 32.

30 Zitiert nach Dieter Langewiesche, Liberalismus in Deutschland, Frankfurt a.M. 1988, S. 128. 
nierten Deutschen Kaiserreich nun auch eine breit entwickelte konfessionelle Vereinsbewegung; die Katholiken als Minderheit beispielsweise bildeten in nahezu jeder Stadt ein stabiles, mithin parteipolitisch relevantes $\mathrm{Milieu}^{31}$. In den nach 1870/71 massenhaft gegründeten Krieger- und Traditionsvereinen schließlich wurden die Reichseinigungskriege in einer Weise zelebriert, daß der in ihnen virulente, manchmal theatralisch-dekorative Militarismus nur als übersteigert bezeichnet werden kann. Dieses merklich veränderte Selbstverständnis fand in vielen Vereinsorganisationen seinen dinglichen Ausdruck. Zur selbstbewußten öffentlichen Darstellung ihres Selbstverständnisses rüsteten sich die Vereine mit Fahnen aus, die in eindeutiger Weise Aussagen über geistige, kulturelle und politische Strömungen einer sich ausdifferenzierenden - nicht nur städtischen - Gesellschaft zulassen. Gewiß, es hatte vereinzelt im Vormärz, während der Revolution von $1848 / 49$, dann wieder gelegentlich während der Reichseinigungskriege Vereine gegeben, die sich schon mit Fahnen ausgerüstet hatten. Aber erst ab 1870/71 vollzog sich eine durchschlagende Veränderung. Durch Ausbildung sozialdemokratischer Vorfeldorganisationen, durch die Entstehung enger katholischer Sozialmilieus in den Städten im Gefolge des Kulturkampfes mit vielen Vereinen und schließlich durch die Begründung zahlloser Militär-, Regiments- und Veteranenvereine im Gefolge der prestigeträchtigen Reichseinigungskriege differenzierte sich das Vereinswesen. Nun, in diesem ausdifferenzierten Zustand, verstärkte sich das Verlangen der Vereine nach spezifischen Symbolen, nach Fahnen und nach Abzeichen, denn man wollte sich - ganz in der militärischen Tradition - eben durch Fahnen und Abzeichen voneinander unterscheiden, mit spezifischen Farben und Symbolen Identität stiften und durch die Verleihung von $\mathrm{Ab}$ - und Ehrenzeichen eine Wertordnung ins Zivilleben übertragen, die der militärischen Sphäre entstammte. In der Zeitspanne von circa 1860 bis 1930 entwickelten die Organisationen, also Vereine, Parteien, Verbände, ihre spezifischen Abzeichen. Dieser Vorgang ist kultur-, sozial- und organisationsgeschichtlich trotz des großen wissenschaftlichen Interesses an den Vereinen, ihren Festen und den Formen ihrer Selbstdarstellung - von der Arbeiterbewegung einmal abgesehen - bisher fast nicht erforscht; dieses Urteil gilt allerdings nicht für Symbole allgemein, wohl aber für die Abzeichen. Dabei spiegelt sich gerade in den Abzeichen, wie eine neuere umfangreiche Studie zu Symbolen der österreichischen Arbeiterbewegung nachweist ${ }^{32}$, in sehr komplexer Weise Geschichte. Sozialdemokratische Abzeichen aus den neunziger Jahren des 19. Jahrhunderts präsentierten z.B. »Ideen und Forderungen « dieser Parteirichtung, waren der kurzgefaßte "Ausdruck der politischen Willensbildung, der po-

31 Zum politischen Katholizismus vgl. den Überblick bei Karl-Egon Lönne, Politischer Katholizismus im 19. und 20. Jahrhundert, Frankfurt a.M., S. 151-192.

32 Eine der wenigen Ausnahmen bietet Josef Seiter, .)Blutigrot und silbrig hell...«.. Bild, Symbolik und Agitation der frühen sozialdemokratischen Arbeiterbewegung in Österreich, Wien, Köln 1991; zum Abzeichen vgl. dort bes. S. 101-148. Zu den Symbolen der Arbeiterbewegung vgl. u.a. Gottfried Korff, Rote Fahnen und Tableaux Vivants. Zum Symbolverständnis der deutschen Arbeiterbewegung, in: Studium zur Arbeiterkultur. Beiträge der 2. Arbeitstagung der Kommission »Arbeiterkultur « in der Deutschen Gesellschaft für Volkskunde in Hamburg vom 8. bis 12. Mai 1983. Hrsg. von Albrecht Lehmann, Münster 1984 (= Beiträge zur Volkskultur in Nordwestdeutschland, Heft 44), S. 103-140. Einen guten Einstieg in die Geschichte der Arbeiterbewegungskultur bietet: Fahnen, Fäuste, Körper. Symbolik und Kultur der Arbeiterbewegung. Hrsg. von Dietmar Petzina, Essen 1986. 
litischen Hoffnungen «. »An die Festtagskleidung gesteckt, firmierten die Embleme auch öffentlich außerhalb der Fest- und Parteiveranstaltungen sozialdemokratische Gesinnung. Aber die verschiedenen Abzeichen auf einem Rock zeigten auch die Multifunktionalität der Parteimitglieder und Funktionäre.«

Die lokale Industrie reagierte auf diese Veränderungen und den sich daraus ergebenden Anpassungsdruck in der deutschen Gesellschaft mit einem immensen Wachstumsschub. Dabei hatte jedoch Preußen seine Hegemonialstellung im kontinentalen Europa überhaupt noch nicht gesichert. Massive Zollrestriktionen und die in den sechziger Jahren Europa in Unruhe versetzenden Nationalbewegungen führten in jener Zeit dazu, daß »Firmen erliegen mußten und viele in den Vorjahren " aus dem Lüdenscheider Umland "zugezogene Arbeiterfamilien, mit Aufopferung ihrer ganzen Habe zu den Eisenbahnorten weiterziehen mußten «. Im Bereich der »Militär-Montirungs-Effekten, Uniformknöpfe, Gürtelschlösser, Helmbeschläge, Beamten-Abzeichen, Kocarden« produzierten einige Lüdenscheider Firmen 1868 aber immerhin Waren im Werte von 150000 Talern $^{33}$. Vieles schien für die Lüdenscheider Industrie noch schwierig und regelungsbedürftig, da trat ein Ereignis von besonderer Tragweite ein. Der Krieg gegen Frankreich 1870 hatte nämlich für die Lüdenscheider Unternehmer zweifelsohne ökonomisch außergewöhnlich positive Folgen. Der Sieg des preußisch bestimmten Militärstaates bewirkte, "daß die [zuvor marktführenden, E.T.] französischen Producenten in Folge der politischen und sozialen Zerrüttung ihres Staatswesens den ungeheuren Weltbedarf nur noch unzuverlässig decken « konnten, "also diese schwierigste Concurrenz durch anderweitige Umstände verdrängt« wurde. Aus diesem Grund häuften sich in Lüdenscheid »die Aufträge aus allen Welttheilen derart massenhaft« an, »daß nur ein verhältnismäßig kleiner Bruchtheil der Anforderungen zu befriedigen möglich « war und »die 10fachen Kräfte» des "Dampfbetriebes und die doppelte Arbeiterzahl« hätten beschäftigt werden können, wenn dem nicht infrastrukturelle Gründe entgegengestanden hätten. Die Lüdenscheider Fabrikanten hofften jedoch, nach deren Beseitigung den "durch die Brachlegung der Pariser Concurrenz« eingetretenen Vorteil »als einen dauernden verzeichnen« zu können. Dies galt natürlich auch für alle speziell für das Militär produzierten Artikel ${ }^{34}$.

Bereits 1872 wurde immer deutlicher, wie sich der Krieg tatsächlich ausgewirkt hatte. Produzierte man im Bereich der »Metall- u. Phantasie-Knöpfe, Broschen, Agraffen, Vorsteck-Nadeln, Gürtelschlösser, Hosenträger-Garnituren, Schieber« etc. gegenüber 1866 in Lüdenscheid Waren statt im Wert von 550000 nun im Wert von 667000 Talern, so hatte sich bei den »Militär-Montirungs-Effecten, Uniformknöpfen, Helmbeschlägen, Beamten-Abzeichen, Cocarden « etc. der Umsatz mehr als verdoppelt (1866: 150000 Taler; 1872: 350000 Taler) ${ }^{35}$. Dieser Vorgang war einerseits von einer Firmengründungswelle begleitet, auf die wir noch eingehen werden, andererseits aber wegen der nunmehr massiven Konkurrenz auch von stark gefallenen Preisen bestimmt. Das lange Zeit herbeigesehnte

33 Jahres-Bericht der Handels-Kammer des Kreises Altena in Lüdenscheid pro 1868/69, Lüdenscheid 1869, S. 72-75.

34 Jahres-Bericht der Handels-Kammer des Kreises Altena in Lüdenscheid pro 1870, Lüdenscheid 1870, S. 101-106.

35 Jahres-Bericht der Handels-Kammer des Kreises Altena in Lüdenscheid pro 1872, Lüdenscheid 1872, S. 54-56. 
und schließlich auch eingeführte Submissionssystem "mit seiner principiellen Berücksichtigung des Mindestfordernden, wie es die Truppenverwaltung adoptirt hat, nachdem man ihr durch Kriegsministerial-Erlass die frühere freie Wahl unter den drei niedrigsten Offerten genommen, treibt unwiderstehlich zur Annahme der Devise sbillig und schlecht « ${ }^{36}$. Aus diesem Grunde baten die Lüdenscheider Fabrikanten um beschränkte Submission, da dadurch bei Einhaltung von Qualitätsstandards Mindestpreise garantiert würden ${ }^{37}$.

Die ausgehenden siebziger und die achtziger Jahre waren für die wirtschaftliche Entwicklung der lokalen Industrie überaus erfolgreich. »Infolge des flotten Geschäftsganges" hatten sich daher "viele neue Firmen etabliert", stellte die Handelskammer Lüdenscheid in ihrem Jahresbericht für 1885 fest $^{38}$. Es gab zwar in den Jahren der Reichsgründung einige bereits seit Jahrzehnten in der Militärknopf- und Militäreffektenproduktion etablierte Firmen, doch lassen sich etwa 30 Firmen benennen, die sich gleichsam als $>$ Trendsetter < betätigten, indem sie sich circa zwischen 1877 und 1887 umstrukturierten oder gar erst gründeten.

Unabhängig von der Beantwortung der Fragen, ob man mit Nennung dieser 30 Firmen schon den Anspruch auf Vollständigkeit erheben darf und wie hoch der Anteil der Militärgeschäfte an ihrem Gesamtumsatz war, kann doch festgehalten werden, daß Lüdenscheid einen Gründerboom erlebte, der im Hinblick auf den spezifischen Charakter, den die lokale Industriestruktur erhielt, Züge des historisch Singulären aufweist. Diese Stadt war nun für den Militarismus, für gesellschaftliche Uniformierungstrends sowie für die Beschaffungswünsche von Militärdienststellen im In- und Ausland zum zentralen Ort geworden, der mit seinen Firmen den vielschichtigen Bedarf gebündelt und hochprofessionell befriedigte. Hier bereits wird deutlich, daß in Lüdenscheid als einer Hochburg der deutschen metallverarbeitenden Kleinteilindustrie nur deshalb außergewöhnliches und weitgehend permanentes Wirtschaftswachstum einsetzte, weil die Industriellen ihre Produktion an den gesteigerten Armeebedarf und an die ab 1870 stark modifizierten, vielfach hypertrophen Formen des sozialen Militarismus koppelten.

Interessant an der beschriebenen Gesamtentwicklung ist, daß sich in Lüdenscheid trotz gelegentlicher, modebedingter Einbrüche ein kontinuierlicher Wachstumsprozeß vollzog, der quer zum >Normalverlauf $\triangleleft$ der Konjunkturentwicklung im Deutschen Kaiserreich stand. In der Phase von 1869 bis 1873, in der Zeit des sogenannten Gründerbooms, verhielten sich die Lüdenscheider Unternehmer eher abwartend. Während der 'großen Depression` zwischen 1873 und 1879 vollzog sich der oben beschriebene, nicht zu wirtschaftlichen, sondern zu gesellschaftlichen Strömungen parallel verlaufende Wachstumsvorgang. Der kontinentale Militarisierungsproze $B$ und die hypertrophe Militarisierung in Deutschland, die Tendenz zur sich verstärkenden Hierarchisierung und Differenzierung in der deutschen Gesellschaft durch Abzeichen, Embleme und Symbole - dies alles setzte im Gefolge der drei Kriege zwischen 1864 und 1870 nach

Jahres-Bericht der Handels-Kammer zu Lüdenscheid pro 1877, Lüdenscheid 1877, S. $24 \mathrm{f}$.

37 Vgl. auch den Jahres-Bericht der Handels-Kammer zu Lüdenscheid pro 1879, Lüdenscheid 1879, S. 25, mit dem Hinweis auf die gefallenen Preise »in Folge der vermehrten Concurrenz«.

38 Jahres-Bericht der Handels-Kammer zu Lüdenscheid für 1885, Lüdenscheid 1885, S. $9 \mathrm{f}$. 
der mit militärischen Mitteln herbeigeführten Begründung des Deutschen Kaiserreichs ein. Seit circa 1885 wurden die Konsequenzen dieses Vorganges für die Lüdenscheider Unternehmer wirtschaftlich handlungsleitend ${ }^{39}$.

Da bei der Produktion von Metall- und Phantasieknöpfen modebedingt 1886 in Lüdenscheid ein Abwärtstrend einsetzte, der überdies durch problematische Zollverhältnisse mit einer Vielzahl von Ländern noch verstärkt wurde, hoffte man auf den baldigen Abschluß von exakten Handelsverträgen. Positiv gestaltete sich 1887 die wirtschaftliche Entwicklung allein in dem hier besonders interessierenden Produktsegment: "In Militär-Montirungs-Effecten war das Geschäft ein recht lebhaftes, da der Bedarf in Folge der Vermehrung der deutschen Armee und der Neuerungen, die bei verschiedenen Ausrüstungsgegenständen getroffen wurden, ein bedeutender war ${ }^{40}$. « Dies sollte sich im Verlauf der Geschichte des Deutschen Kaiserreiches, wie noch zu zeigen sein wird, fortsetzen. Die Friedenspräsenzstärke stieg von 1870 mit rund 400000 Mann auf 434000 Mann im Jahre 1880, auf 509000 im Jahre 1890, auf 629000 im Jahre 1900 bis auf die gegenüber dem Gründungsjahr des Kaiserreiches mehr als verdoppelte Zahl von 864000 Mann im Jahr 1913 ${ }^{41}$. Daher war es aus unternehmerischer Sicht nur konsequent, wenn man frühzeitig aus dem Ende der achtziger Jahre tatsächlich oder vermeintlich immer problematischer werdenden Metallknopfgeschäft auf dem modeabhängigen Bekleidungssektor in den Militärbereich umsattelte. Die Lüdenscheider Handelskammer registrierte diese Entwicklung und brachte sie auf den Punkt: "Verschiedene Fabrikanten von Modeknöpfen versuchten in der Herstellung von Militär- und Beamtenknöpfen, Tornistergarnituren, Helmbeschlägen und ähnlichen Artikeln für den Armeebedarf [...] ihren Arbeitern Beschäftigung zu geben.« Und die Berichte wiederholten sich. Immer mehr Lüdenscheider Unternehmer würden auf dem Markt für »Militär-Montirungs-Effecten, Uniformknöpfe, Livreeknöpfe« Fuß zu fassen suchen, denn bei den "umfangreichen Neuanschaffungen für die Armee war der Geschäftsgang« auch 1888 in diesem Bereich äußerst lebhaft. Die Lüdenscheider Handelskammer beobachtete diese Tendenz: "Den für diese Branche speciell eingerichteten Betrieben wurde durch [...] die Modeknopffabrikanten, die auf diesem Gebiete für ihre Arbeiter in der flauen Zeit um jeden Preis Beschäftigung suchten, die empfindlichste Concurrenz gemacht. « Auch der Absatz an "Abzeichen, Cocarden, Verzierungen für Hüte und Mützen etc.«, der »sich fast ausschließlich auf das Inland «, vielfach auf den Verkauf an Vereinsorganisationen beschränkte, befand sich 1888 durch die große "Mannigfaltigkeit" der Muster in einem positiven Entwicklungstrend ${ }^{42}$.

Hostert, Entwicklung (wie Anm. 15), S. 179-202, zur Chronologie der Firmengründungen in Lüdenscheid.

Jahres-Bericht der Handels-Kammer zu Lüdenscheid für 1887, Lüdenscheid 1887, S. 22; die Zollfragen waren für die Unternehmer so gewichtig daß sie sich mehrfach bei den zuständigen Ministerien in Berlin beschwerten. Am 9.10.1888 kam zur Untersuchung der "Produktions- und Handelsverhältnisse der niederrheinisch-westfälischen Metallknopffabrikation [...] der vortragende Rath im Handelsministerium Herr Geheimer Ober-Regierungsrath Mosler « nach Lüdenscheid, wo er sich mit Vertretern der Kammer zu Gesprächen einfand. Vgl. Jahres-Bericht der Handels-Kammer zu Lüdenscheid für 1888, Lüdenscheid 1888, S. 9. Zu den allgemeinen Zoll- und Absatzbedingungen für die Lüdenscheider Fabrikanten vgl. Romland, Entwicklung (wie Anm. 20), S. 26-31.

1 Zahlen nach Wehler, Kaiserreich (wie Anm. 28), S. 151.

2 Jahresbericht der Handelskammer zu Lüdenscheid für 1888, Lüdenscheid 1888, 
Wiederholt kam es nun vor, daß seitens der Militärverwaltungen Sonderwünsche geäußert wurden, wodurch "grosse Aufträge" bei den Lüdenscheider Firmen eingingen, so etwa bei »Patronentaschen-Federeinsätzen, Tornisternadeln, Carabiner- und Brotbeutelhaken usw.«. Auch das »Lieferungsgeschäft in Knöpfen für Militär-, Post- und Eisenbahnbeamte « lief zum Ausgang der achtziger Jahre in Lüdenscheid auf dem mittlerweile gewohnt hohen Niveau ${ }^{43}$. Diese Stadt, das läßt sich zusammenfassen, hatte zwischen 1870 und 1890 einen Umstrukturierungsprozeß erfahren, der den in der Überschrift des vorliegenden Beitrages angedeuteten Strukturzusammenhang in geradezu beispielhafter Weise erkennbar werden ließ. Natürlich bot auch der Auslandsmarkt den Produzenten von Militäreffekten und Militärknöpfen ein weites Betätigungsfeld ${ }^{44}$. Denn auch das europäische und außereuropäische Ausland erlebte - wie dies in seinen Konsequenzen für die Lüdenscheider Industrie nachgewiesen werden $k a n n^{45}$ - im 19. Jahrhundert einen Militarisierungsprozeß bedeutenden Ausmaßes.

\section{Das Aluminium und das Militär}

»Das Jahr 1891 « brachte der Lüdenscheider Industrie »die Einführung des Aluminiums zur Massenverarbeitung«. Der bedeutende Lüdenscheider Unternehmer Carl Berg ${ }^{46}$ hatte angeregt, »die Beschläge der Mannschaftszelte des deutschen Heeres aus Aluminium herzustellen; seine Fabrik in Lüdenscheid erhielt den Auftrag, 1 Million Garnituren anzufertigen. Wenige Jahre später führte das preußische Kriegsministerium Feldflaschen und Kochgeschirre nach Bergschen Mustern ein ${ }^{47}$.« Diese für den Militärbedarf bahnbrechenden Innovationen wur-

S. 23 f.; die Klage über das Ausweichen von Modeknopffabrikanten auf den Militärund Abzeichenbereich wiederholte sich nun regelmäßig: "Größere Lieferungen, sonst durchweg in festen Händen, welche diesen Fabrikationszweig als Specialität betrieben, sind von Fabrikanten verwandter Branchen übernommen worden, die um jeden Preis ihren Betrieb aufrecht erhalten, resp. ihren Arbeitern Beschäftigung gewähren wollten."So auch im Jahresbericht der Handelskammer zu Lüdenscheid für 1889, Lüdenscheid 1889, S. 18. Das Problem des Zeichen- und Musterschutzes wird thematisiert im Jahresbericht der Handelskammer zu Lüdenscheid für 1892, Lüdenscheid 1892, S. 19.

43 Jahresbericht der Handelskammer zu Lüdenscheid für 1890, Lüdenscheid 1890, S. 22.

44 Vgl. Bernd D. Plaum, »Fliegt in alle Länder und empfehlt uns!« Bedingungsfaktoren des wirtschaftlichen Erfolges und Absatzstrategien der bergisch-märkischen Metallknopfindustrie, in: "Fliegt in alle Länder und empfehlt uns!« - Gesellschafts- und wirtschaftsgeschichtliche Studien zur Lüdenscheider und Wuppertäler Metallknopfund Abzeichenindustrie im 19. und 20. Jahrhundert. Hrsg. von Eckhard Trox, Lüdenscheid 1996 (= Forschungen zur Geschichte Preußens im südlichen Westfalen, Bd 2), S. 23-39.

45 Ein gutes Beispiel bietet Walter Hostert, Lüdenscheider Knöpfe für den Zaren. Das Majestätswappen des zaristischen Rußland, in: Der Reidemeister, 94 (1985), S. 741-756. Eine Quantifizierung der Exporte ist allerdings beim gegenwärtigen Kenntnisstand nicht möglich.

46 Kurzbiographie des Begründers der deutschen Aluminiumindustrie mit weiterführender Literatur bei Eckhard Trox, Art. Carl Berg, in: Porträts (wie Anm. 15), S. 47.

47 Hostert, Entwicklung (wie Anm. 15), S. 120; vgl. auch Fritz Grüber, Die Entwicklung 
den natürlich auch von der Lüdenscheider Handelskammer herausgestrichen: "Nachdem das Aluminium für verschiedene Ausrüstungsgegenstände der deutschen Armee eine immer weitergehende Verwendung gefunden, war ein hiesiges Werk « - gemeint ist das von Carl Berg — »in dieser Branche flott beschäftigt und konnte mehreren hundert Arbeitern, die früher vorwiegend in den Knopffabriken tätig waren, Verdienst geboten werden ${ }^{48}$." Damit wird deutlich, daß erneut ein herausragender Lüdenscheider Unternehmer sich entschieden hatte, in den neunziger Jahren seine Firma möglichst wenig den Schwankungen der Mode auszusetzen, sondern darauf zu vertrauen, daß die Produktion für den Militärbedarf konstant Profit abwerfen würde. Die Herstellung "verschiedener Artikel aus Aluminium für Armeebedarf « nahm in dieser Lüdenscheider Firma in den folgenden Jahren einen immer größeren Umfang $a{ }^{49}$.

Die lokalen Fabrikanten, die sich auf Militärmontierungseffekten, Zivil- und Militäruniformknöpfe spezialisiert hatten, waren zwar in den letzten Jahren vor der Jahrhundertwende an Geschäften mit »den Armee- und Marine-, sowie den Eisenbahnverwaltungen in hervorragendem Maße beteiligt « ${ }^{50}$ bzw. konnten bei "dem grossen Bedarf« an diesen Artikeln »wiederum namhafte Aufträge« verbuchen $^{51}$. Aber auch auf diesem Gebiet entwickelte die Firma Berg allmählich eine herausragende Position. "Vielfach war die Firma « sogar "als Mustermacherin für das Preußische Kriegsministerium tätig, und im Jahre 1907 durfte« diese durch die zahlreichen Aluminiumerzeugnisse mit dem Kriegsministerium eng zusammenarbeitende Firma "den ganzen Bedarf von mehreren Millionen neuen Knöpfen für die feldgrauen Uniformen des preußischen Heeres mit Ausnahme des Gardekorps « herstellen. Auch die beim Heere bis 1918 sich »in Gebrauch befindlichen Helmbeschläge aus Aluminiumbronze« wurden von der Firma Berg hergestellt. Diese Firma stieg auch in das Abzeichengeschäft ein. In den neunziger Jahren des 19. Jahrhunderts gingen Kochgeschirre, Feldflaschen und Trinkbecher aus Aluminium in Serie. Die Kameradschafts-Kochapparate aus Aluminium « wurden von der deutschen Artillerie angenommen. Als im Zusammenhang mit dem Boxeraufstand im Jahre 1900 zur Sicherung des deutschen Einflusses eine Interventionstruppe unter Graf Waldersee nach China entsandt wurde, über-

der Lüdenscheider Aluminiumindustrie. Eine kritische betriebswirtschaftliche Studie, Lüdenscheid 1925. Allgemein zum Aluminium vgl. Aluminium. Die ersten hundert Jahre. Hrsg. von Hans Joliet, Düsseldorf 1988, und Aluminium. Das Metall der Moderne. Gestalt - Gebrauch - Geschichte. Hrsg. von Werner Schäfke, Thomas Schleper und Max Tauch, Köln 1991. Auf die unternehmerische Leistung von Carl Berg hat, eingebettet in eine kurze Industriegeschichte der Innovationen des märkischen Sauerlandes, neuerdings noch einmal Bernd D. Plaum in einem vorzüglichen Beitrag anläßlich des 150. Jahrestags der Gründung der Südwestfälischen Industrie- und Handelskammer hingewiesen. Vgl. hierzu ders., Durch Innovationen Schritt halten. Ein Beitrag zur Industriegeschichte des märkischen Sauerlandes 1844-1994, in: Innovationen 1844-1994. Hrsg. von der Südwestfälischen Industrie- und Handelskammer zu Hagen. Ausstellungskatalog, Hagen 1994, S. 19-52, bes. S. 45-47. bis 1897; Zitat aus dem Bericht von 1893, S. 17. Prognose nach dem Jahresbericht der Handelskammer zu Lüdenscheid für 1904/05, Lüdenscheid 1905, S. 27.

50 Jahresbericht der Handelskammer zu Lüdenscheid für 1896/97, Lüdenscheid 1897, S. 26.

51 Jahresbericht der Handelskammer zu Lüdenscheid für 1898/99, Lüdenscheid 1899, S. 27. 
trug das Kriegsministerium der Firma Berg die "Ausrüstung der ostasiatischen Expedition mit 40000 Kochgeschirren «. »Dieses Kochgeschirr bestand seine Feuerprobe in dem Feldzuge so gut«, daß nach dem Ersten Weltkrieg »ein ganz ähnliches Modell als Armeefeldkessel für das ganze deutsche Heer" eingeführt wurde. Die Firma genoß nun Weltgeltung, so daß »Bergs Feldflaschen und Kochgeschirre bald "zu Hunderttausenden auf beiden Seiten in allen großen Kriegen der letzten Jahrzehnte in Gebrauch waren " und insbesondere auch im Ersten Weltkrieg "große Dienste" leisteten. Denn: »Daß die Firma, die schon in Friedenszeiten zum großen Teil für den Heeresbedarf tätig war, mit Leichtigkeit sich schnell den Bedürfnissen der Heeresleitung in dem großen Weltkriege anzupassen wußte, ist selbstverständlich und braucht wohl nicht besonders ausgeführt zu werden ${ }^{52}$." Jenes Zitat macht noch einmal deutlich, daß Unternehmen wie das Bergsche immer auch den ausländischen Bedarf an Militärprodukten befriedigten. Wenn Berg auch 1916 nicht an französische Militärdienststellen lieferte, so kannte angesichts der weltweit wachsenden Armeen das Geschäft mit kleinteiligem Militärbedarf und Objekten des dekorativen Militarismus doch kaum Grenzen. Mancher als mittelständisch zu charakterisierende Unternehmer erwirtschaftete gerade im ausgehenden 19. und beginnenden 20. Jahrhundert viele Millionen Reichsmark Gewinn.

Der außergewöhnliche Ruf der Firma Berg gründete sich schließlich auch darauf, daß durch risikofreudigen Kapitaleinsatz des.Kommerzienrates Carl Berg auf dem Lüdenscheider Firmengelände die ersten Luftschiffe vormontiert wurden. Berg hatte durch seine Ingenieure dem Erfinder des ersten lenkbaren Luftschiffes nach dem starren System, David Schwarz, bei der genauen Erstellung der Konstruktionspläne Schützenhilfe geleistet und ihm überdies kostenlos das Aluminium überlassen. Auch dem Grafen Zeppelin half Carl Berg.

\section{Die Vorbereitungen für den Mobilmachungsfall}

Galt Bismarck lange Jahre bis zu seinem durch Wilhelm II. herbeigeführten Sturz als Garant einer europäischen Friedensordnung, so schürten die säbelrasselnden Reden des neuen Kaisers ab 1890 in Europa Kriegsängste, von denen auch eine Industrie nicht unberührt bleiben konnte, die ihre Entwicklung in einem so bedeutenden Maße an die Befriedigung des Militärbedarfs und an die Trends und Denkmuster einer militarisierten Gesellschaft gebunden hatte.

Ein Staat, der sich 1913 gleichsam als vorläufig letzten Höhepunkt die smega‘-militaristische Zabern-Affäre leistete ${ }^{53}$, war auf eine zuverlässige Industrie

52 Alle Zitate nach einem undatierten, wahrscheinlich aus der Zeit kurz nach dem Ersten Weltkrieg stammenden maschinenschriftlichen Text von Heinz Nöthe, Wilhelm Berg, Lüdenscheid, in: Stadtarchiv Lüdenscheid, C 995.

53 Kurz dargestellt und in die Geschichte der mneuartige[n] Qualität des deutschen Militarismus" nach den »drei Einigungskriegen« eingeordnet bei Hans-Ulrich Wehler, Deutsche Gesellschaftsgeschichte. Bd 3: Von der »Deutschen Doppelrevolution « bis zum Beginn des Ersten Weltkrieges 1849-1914, München 1995, S. 1125-1129. Wehlers zentrale Feststellung - in »keiner westlichen Gesellschaft besetzte das Militär eine so 
angewiesen, die sich im Mobilmachungsfall zur sofortigen Umstellung ihrer Produktion auf Rüstungs- und Kriegsbedarf in der Lage sah. Die. Lüdenscheider Fabrikanten hatten das veränderte, von außenpolitischen Turbulenzen geprägte Klima im Kaiserreich erfaßt und sich auf die Situation eingestellt. In der Stadt begann man nun bald, insbesondere mit dem Ausbruch des Weltkrieges, auch Drähte für die Feldkabel und Patronenhülsen herzustellen. Doch soll es hier abermals nicht um die Rüstungsindustrie im engeren Sinn, sondern um den oben näher charakterisierten dekorativen Militarismus gehen. Als die Lüdenscheider Handelskammer 1901 durch "Vermittelung des Herrn Regierungspräsidenten " vom »Königl. Bekleidungsamte in Kassel aufgefordert wurde, über die Leistungsfähigkeit der [...] bestehenden Fabriken in Militärknöpfen, Beschlägen und andern Ausrüstungsgegenständen zu berichten", war ihr die Darstellung der im Mobilisierungsfall freiwerdenden Kapazitäten besonders wichtig:

»Neben Militärknöpfen werden hier namentlich Modeknöpfe für Damenkleider, sowie Manschettenknöpfe, Metallknöpfe für Knabenkleider und MetallHosenknöpfe - letztere besonders in ganz bedeutenden Posten - fabriziert. Eine Mobilmachung hat für diese Fabrikationszweige eine Stockung des Betriebes zur Folge und die frei werdenden geschulten Arbeitskräfte und Maschinen stehen dann den Fabrikanten von Militärartikeln zur Verfügung. Dazu kommt noch, daß aus einer Reihe von verwandten Fabrikationszweigen der Metallkurzwarenbranche, wir nennen die Schnallenfabrikation, die Fabrikation von Abzeichen und Beschlägen aller Art u.s.w., in gleicher Weise Arbeiter, Maschinen und Betriebskraft den Fabrikanten von Militärartikeln im Falle einer Mobilmachung zu Gebote stehen."

Nach dem Resümee der Handelskammer konnten also alle Fabrikanten, die in Lüdenscheid Metallwaren produzierten, in der Stunde `nationaler Gefahr ‘ »ihre gesamten eigenen Arbeitskräfte und Maschinen bei forciertem Betrieb in den Dienst der Militärlieferungen " stellen ${ }^{54}$. Dieser Tonfall wurde von der Kammer immer wieder angeschlagen. Insbesondere sollten die Militärbehörden - gleichgültig, ob in wirtschaftlichen Krisen- oder allgemein in Friedenszeiten - darauf bedacht sein, eine »regelmäßige Beschäftigung der alten, bewährten Lieferanten sicherzustellen, um im Fall der Mobilmachung « auf jene zurückgreifen zu können $^{55}$. Das galt natürlich insbesondere für diejenigen Fabriken, die kriegswichtige Artikel produzierten, aber man wollte im Mobilmachungsfall unbedingt auch mit den Spezialartikeln, namentlich den »Militär-Montierungseffekten«, im Geschäft bleiben. Man wies seitens der Handelskammer daher auch noch einmal in aller Deutlichkeit darauf hin, daß die "Leistungsfähigkeit der hiesigen Fabriken [...] sowohl qualitativ als quantitativ eine solche« sei, daß die Firmen "bei Feststellung derselben, wie sie u.a. periodisch seitens der Militär-Behörden « erfolgte, insbesondere wegen der jahrzehntelangen Tradition und der hervorragenden

hohe Position in der Prestigehierarchie wie in Deutschland, nirgendwo sonst übte der Sozialmilitarismus einen so tiefgreifenden, dauerhaften Einfluß aus« (ebd., S. 1126) wird von mir geteilt. Wehlers Statement wird m.E. durch die in diesem Beitrag vorgestellten Thesen bestätigt.

54 Jahresbericht der Handelskammer zu Lüdenscheid für 1901/1902, Lüdenscheid 1902, S. 19.

55 Jahresbericht der Handelskammer zu Lüdenscheid für 1902/1903, Lüdenscheid 1903, S. 22. 
Facharbeiter, Graveurmeister etc. bei den Militäreffekten »an erster Stelle figurieren werden ${ }^{56}$.

$\mathrm{Zu}$ Ängsten um die wirtschaftlich-industrielle Zukunft Lüdenscheids bestand in der Tat während des Deutschen Kaiserreichs, vor allem zu Beginn des 20. Jahrhunderts keinerlei Anlaß. Die Handelskammer - immer bedacht, im Interesse der von ihr vertretenen Unternehmen die wirtschaftlichen Verhältnisse als schwierig, die Unternehmensgewinne als zu gering und die Konkurrenz als drückend zu bewerten - stellte für das Geschäftsjahr 1906 fest, daß »MilitärMontirungs-Effekten aus Kupferlegierungen, Uniformknöpfe, Livreeknöpfe, Metallabzeichen, Helmbeschläge aus Messing und Aluminiumbronze« etc. die Vollbeschäftigung bei den zahlreichen Lüdenscheider Spezialbetrieben »fast unausgesetzt « sicherstellten. Und: »An den Lieferungen für Heer und Marine blieben die hiesigen Fabrikanten in hervorragendem Maße beteiligt. " Die Überlegungen zum Mobilmachungsfall wurden von einigen Unternehmern durch einen geradezu überbordenden Patriotismus flankiert. Das war angesichts des preußisch-protestantischen und konservativ-nationalen Milieus, in denen viele dieser Unternehmer ihre politische Sozialisation erfuhren, auch wenig verwunderlich. Einige lokale Firmen betätigten sich aus eigener Initiative als Großprotagonisten der Militärmonarchie. Als Kaiser Wilhelm II. 1906 auf ein 25jähriges Eheleben mit Auguste Viktoria zurückblickte, nahm die Firma "Steinhauer \& Lück. Vereinsabzeichen-Fabrik « dies zum Anlaß, mit Sonderprodukten über ein gedrucktes Werbeblatt an die Öffentlichkeit $\mathrm{zu}$ treten. Auf diesem wurden Vorsteck- und Krawattennadeln, Broschen, Ständer, Medaillen und Manschettenknöpfe angeboten. Sie zeigten das floral oder mit Perlenbesatz geschmückte, z.T. auch bekrönte "Jubelpaar « mit dem stets uniformierten und mit Orden dekorierten Wilhelm II., dessen Geschmack mit der Kampagne der Lüdenscheider Firma voll getroffen worden sein dürfte. Steinhauer \& Lück warben mit folgendem Text:

"Am 27. Februar 1906 findet die 25jährige Jubelfeier (silberne Hochzeit) unseres allverehrten und geliebten Kaiserpaares statt. Dieser Jubeltag wird doch aller Wahrscheinlichkeit nach durch Schulfeiern festlich begangen. - Um nun der Schuljugend die hohe Bedeutung dieses Tages so recht ans Herz zu legen und die echten patriotischen Gefühle zu unserm hochverehrten Kaiserpaare zu beleben, haben wir auf Veranlassung befreundeter Lehrerkreise kleine Andenken [...] angefertigt und die Preise so billig gestellt, daß es auch den weniger bemittelten Kindern möglich ist, ein derartiges Andenken anschaffen zu können.«

Man bat »höflichst die Herren Lehrer, falls im dortigen Schulbezirk Schulfeiern veranstaltet werden sollten, die Anschaffung solcher Andenken gütigst befürworten und diesen illustrierten Katalog bei den Herren Kollegen zirkulieren lassen zu wollen. « Die Firma meinte es ernst mit dem "Jubelfest « und wollte ihr kleines nationales Scherflein dazu beitragen: »Bei jedem 100 Stück geben wir für unbemittelte Kinder 8 Stück von derselben Art gratis. « Der `schwarze Lehrplan`, das pädagogische Programm dieser Aktion, kam am gültigsten auf einer der angebotenen Medaillen zum Ausdruck. Auf der Vorderseite erkennt man in der Mitte das Kaiserpaar, oben die Kaiserkrone, unten den Preußenadler. Der Kaiser zeigt sich mit preußischem Paradehelm und Uniform. Die Medaille mit dem 
sherrlichen Anblick ‘ auf der Vorderseite hatte natürlich eine Kehrseite, auf der sich eine wehrhafte Borussia und das für die Regierungszeit Wilhelm II. unverzichtbare Schlachtschiff befand. Kaiserreich, Kaiserpaar, militärischer Geist, Schlachtflottenbau, Weltgeltung auf den Meeren und deutsche Weltmachtstellung überhaupt - das sollte den Schülern auch anläßlich des kaiserlichen Ehejubiläums als zusammengehörig in Erinnerung gebracht werden ${ }^{57}$.

Kurz vor dem Ausbruch des Ersten Weltkrieges kam jedoch ein neuer, zunächst leiser, aber unüberhörbarer Tonfall in die Argumentation der Lüdenscheider Handelskammer, die als ein Sprachrohr namentlich der für den Armeebedarf produzierenden Firmen fungierte. Es zeichnete sich ein Umdenken ab. Bei »einzelnen Militärartikeln«, zum Beispiel bei so auffällig schmückend-dysfunktionalen wie Helmbeschlägen, waren nämlich die »Heeresverwaltungen bestrebt «, bei der wachsenden Kritik der Opposition an den hypertrophen Erscheinungen des Militarismus zunächst einmal "alles Glänzende von den Uniformstücken fern zu halten«. Aus heutiger Sicht könnte man geneigt sein, in einer solchen Formulierung aus dem Jahre 1912 - zumindest mit Blick auf die bis dahin in Deutschland vorhandenen Traditionsstränge - eine ansatzweise Vorwegnahme der in der Weimarer Republik staatlicherseits praktizierten ordens- und auszeichnungsfeindlichen Politik zu sehen, die eine Ablehnung übersteigerter Erscheinungen des Militarismus immer mit einschloß. Das bedeutete aber nicht, daß die Unternehmen während des Ersten Weltkrieges weniger Umsatz gemacht hätten. Die Firma Friedrich Linden verzeichnete "mit Kriegsausbruch eine in dem Umfange nie gekannte Beschäftigung in Uniformknöpfen usw. für das deutsche Kriegsheer «. Der »Massenbedarf an Militärknöpfen brachte« - so die Beobachtung eines Zeitzeugen des Ersten Weltkrieges in Barmen, also der zweiten bergisch-märkischen >Knopfhochburg « - eine regelrechte »Hochkonjunktur « mit sich. Barmer Textilbetriebe stellten wegen des Rohstoffmangels auf die Knopfproduktion um. Im Jahre 1916/17 existierten auf einmal "mehr als 110 Barmer Firmen, darunter solche mit mehr als 150 Mann Belegschaft «, die "mit der Herstellung außer sonstigen Militäreffekten auch mit der Fabrikation von Militärknöpfen befaßt « waren ${ }^{58}$.

57 Jahresbericht der Handelskammer zu Lüdenscheid für 1906/1907, Lüdenscheid 1907, S. 29. - Das insgesamt 28 Artikel zu diesem Anlaß anbietende, einseitige Werbeblatt der Firma "Steinhauer \& Lück. Vereinsabzeichen-Fabrik, 25jährige Jubelfeier (silberne Hochzeit), Lüdenscheid 1906«, befindet sich in der Sammlung des Stadtmuseums Lüdenscheid. Zum politisch-sozialen Milieu der Unternehmerschaft in Lüdenscheid vgl. Jean-Luc Malvache, Die Lüdenscheider Arbeiterbewegung. Von der Vereinzelung zum Verein, in: Fahne (wie Anm. 15), S. 11-37, bes. S. 11-14. Jahresbericht der Handelskammer zu Lüdenscheid für 1911/12, Lüdenscheid 1912, S. 31. Eine zeitgenössische Wiedergabe und Bewertung der Rüstungsausgaben des Deutschen Kaiserreichs stammt von Matthias Erzberger, Die Rüstungsausgaben des Deutschen Reichs, Stuttgart 1914. Erzberger gestattet auch einen Einblick in die für Lüdenscheid besonders interessante Position »Bekleidung und Ausrüstung in ihrem Verhältnis zu den Gesamtausgaben. Zum Boom in der Militärknopfbranche während des Ersten Weltkrieges vgl. die Betriebsfestschrift der Firma Friedrich Linden »Ihrem ehrwürdigen Betriebsführer Herrn Ernst Linden zum steten Andenken gewidmet, anläßlich seines 40jährigen Inhaber-Jubiläums am 4. November 1935«, Lüdenscheid 1936, S. 3. Zur Kriegs- und Nachkriegszeit, insbesondere in Barmen, vgl. Erwin Weber, Die Bergisch-Märkische Knopfindustrie. Eine wirtschaftsgeschichtliche Untersuchung, Köln 1924, S. 21-23 und S. 30. Zur Knopfindustrie in Barmen vgl. auch Herbert Pogt, 


\section{Die Folgen des Ersten Weltkrieges und die Weimarer Republik}

»Das deutsche Volk erhielt « am 11. August 1919 "zum ersten Mal in seiner Geschichte eine republikanische und demokratische Verfassung, nachdem der militärischen Niederlage im Ersten Weltkrieg die Novemberrevolution von 1918 gefolgt war und die seit der Reichsgründung von 1871 bestehende", stark die militärischen Traditionen betonende »Staatsorganisation beseitigt hatte «. Die Verfassung der Weimarer Republik beschränkte sich nicht darauf, die alte Militärmonarchie gleichsam von ihrer Spitze her zu zertrümmern, sondern buchstabierte den mehrheitlich von Linksliberalen und Sozialdemokraten geprägten , Weimarer Geist ‘ bis in die einzelnen Artikel hinein durch. Dazu gehörte insbesondere der in Art. 109 geregelte Grundsatz, daß »alle Deutschen [...] vor dem Gesetz gleich “ seien. In diesem Sinne bestimmte der Artikel konsequent weiter: "Orden und Ehrenzeichen dürfen vom Staat nicht verliehen werden. Kein Deutscher darf von ausländischen Regierungen [...] Orden annehmen ${ }^{59}$. « Hitler — so viel sei hier vorweggenommen - war es, der diesen Grundsatz, den die Vereine und Verbände gleichsam von unten schon während der Weimarer Republik durch das vielfach überbordende Auszeichnungs(un)wesen innerlich ausgehöhlt hatten, 1933 in das vollständige Gegenteil verkehrte. Für die hier interessierenden lokalen Firmen wurde die Situation in der sich zum Teil demilitarisierenden Weimarer Republik schwieriger, obwohl sie weiter in Deutschland ihren Marktvorsprung hielten. Zwingend erforderlich waren aber die Intensivierung bestehender oder die Konstituierung neuer organisatorischer Zusammenschlüsse.

Im Vordergrund der organisatorischen Bestrebungen der Knopf-, Militäreffekten- und Abzeichenfabrikanten stand eine Kartellstrategie, um durch Absprachen die großen Preisunterschiede bei den Ausschreibungsergebnissen der Bekleidungsämter für die Armee zu beseitigen und untereinander Gewinnspannen zu vereinbaren, die das unternehmerische Risiko als lohnend erscheinen ließen. Dazu hatte man bereits am 11. November 1899 eine Vereinigung für die Militärknopfbranche begründet, die sich offiziell als »Verband Deutscher Uniformknopffabrikanten « bezeichnete, lediglich ihre Geschäftstelle in Berlin, ihren eigentlichen Sitz aber in Lüdenscheid hatte. Seit 1920 existierte in Barmen ein »Verband der deutschen Celluloid-, Metall- und Stoffknopffabrikanten e.V.«, bereits 1895 hatte sich ein "Verband deutscher Steinnußknopfindustrieller e.V.« in Schmölln gegründet. Diese drei Verbände bildeten zusammen mit drei anderen Berliner Organisationen den "Zentralverband deutscher Knopffabrikanten e.V.« mit Sitz in Berlin. Daß der für die Vertretung der Interessen der Uniformknopffabrikanten zuständige Verband in Lüdenscheid angesiedelt war, kann vor dem Hintergrund der bisher geschilderten Zusammenhänge nicht verwundern ${ }^{60}$. Zu

»Im Zentrum der Knopfindustrie«. Zur Geschichte des Wuppertaler Knopfgewerbes, in: Fliegt in alle Länder (wie Anm. 44), S. 69-86.

59 Die Verfassung des Deutschen Reiches vom 11. August 1919. Hrsg. von Hermann Mosler, Stuttgart 1977; Zitate S. 65 und S. 37.

60 Vgl. auch Rainer Assmann, "Bescheidener Verdienst und auskömmlicher Lohn « Das deutsche Knopfkartell der Uniformknopf-Fabrikanten in Lüdenscheid, in: Fliegt in alle Länder (wie Anm. 44), S. 87-96; zur Liste der Mitglieder dieses Kartells vgl. ebd., S. 95. Vgl. dazu auch das auf den 5.6.1926 datierte Schreiben des "Zentralverbandes Deutscher Knopffabrikanten e.V.«, der seine Geschäftstelle in der Zimmerstr. 3-4 
diesem Verband gehörten einige Lüdenscheider Firmen, die z.T. seit der Jahrhundertwende in Beziehung zum $»$ Reichsverband der Uniform-Lieferungs-Fabrikanten e.V.« standen ${ }^{61}$. Organisatorisch bildete Lüdenscheid offenkundig das Zentrum der deutschen Uniformknopflieferanten.

Das Geschäft der Lüdenscheider Firmen mit Dienststellen des Reichsheeres gestaltete sich trotz der massiven zwangsweisen Truppenreduzierung im Gefolge des Versailler Vertrages auch während der Weimarer Republik durchaus als lohnend. Wegen der Belieferung mit Uniformknöpfen und Effekten für den deutschen Bedarf stand z.B. die Firma Julius Bergmann GmbH mit der "Zentralbeschaffungsstelle für Heer und Marine« in Berlin in Verbindung, die in den einzelnen deutschen Ländern jeweils noch über Landesauftragsstellen verfügte. Im Mai 1926 lieferte diese Firma 32000 "Reichsheerrockknöpfe“. Auch das Auslandgeschäft mit Militärknöpfen florierte noch. Im Oktober 1925 hatte diese Firma einen Auftrag über 280000 Uniformknöpfe eingeworben. Bereits im April 1925 kontaktierte man über das finnische Generalkonsulat in Berlin mit dem Verteidigungsamt in Helsinki, wobei es um einen Auftrag von 2000000 Uniformknöpfen ging ${ }^{62}$. Da seitens des Staates Orden und Ehrenzeichen nicht verliehen werden durften, bemühten sich die Firmen intensiv um den militärisch-zivilen Zwischenmarkt. Spürten sie Informationen über Regimentstage, Traditionsfeiern oder sonstige militärisch-nationale Gedenktage etc. auf, so setzten sie sich mit den Veranstaltern in Verbindung und konnten auf diesem Wege zu einträglichen Aufträgen gelangen ${ }^{63}$. Solche Tage - häufig waren sie mit Erinnerungen an erfolgreiche Schlachten verknüpft - erfreuten sich bei konservativ-militärischen Traditionsverbänden größter Beliebtheit, konnte man doch bei solchen Gelegenheiten die alte Herrlichkeit der preußischen Militärmonarchie wiederauferstehen lassen. Da Gedenktage dieser Art häufig durchgeführt wurden, sicherten sie der Lüdenscheider Abzeichenindustrie gute Einkünfte. Gleichzeitig höhlte das Verleihen ordenähnlicher Abzeichen aber die Bestimmungen der Weimarer Verfassung gleichsam von innen aus.

Einen für zahlreiche vergleichbare Firmen repräsentativen Überblick sowohl über die Angebotspalette der Abzeichenindustrie als auch über die Nachfragestruktur seitens der einschlägigen Organisationen bietet ein Katalog der bedeutenden Lüdenscheider Firma Wilhelm Deumer aus dem Jahre 192664. In diesem Druckwerk finden sich Abzeichen für die Arbeiterbewegung, etwa für den Arbeiterradfahrerbund, für Arbeiterturnvereine, für den Arbeitersängerbund,

in Berlin hatte, an die Firma Brüninghaus \& Co in Lüdenscheid, in: Unverzeichneter Nachlaß der Firma Julius Bergmann GmbH, Lüdenscheid, der in der Sammlung des Stadtmuseums Lüdenscheid aufbewahrt wird; allgemein zur Kartellstruktur bei der Knopfindustrie vgl. Weber, Knopfindustrie (wie Anm. 58), S. $44 \mathrm{f}$.

61 Vgl. dazu den Schriftwechsel der »Eisenbahn-Gruppe im Reichsverband der UniformLieferungs-Fabrikanten e.V.« mit Sitz in Berlin und der Firma Julius Bergmann GmbH vom April 1926, in: Nachlaß (wie Anm. 60).

62 Vgl. den diesbezüglichen Schriftverkehr der Firna Julius Bergmann GmbH mit der »Zentralbeschaffungsstelle für Heer und Marine « vom 8.4.1925 und 11.5.1925, in: ebd.

63 Vgl. den diesbezüglichen Schriftverkehr in: ebd.

64 Firma Wilhelm Deumer GmbH, Abzeichen. Medaillen, Plaketten, Orden, Fahnennägel. In Metall, echter Glasemaille, Galvanoplastik für Vereine, Sport, Beamte, Militär. 1926, Lüdenscheid 1926. Der Katalog enthält 143 bedruckte Seiten mit jeweils zahlreichen Abbildungen. Für den freundlichen Hinweis auf den Deumer-Katalog danke ich Herrn Dr. Hostert. 
für Arbeiterunterstützungsvereine sowie für die diversen konfessionellen Arbeitervereine ${ }^{65}$. Kleine sozialdemokratische Farbtupfer waren neben den Abzeichen für die Arbeitervereine allenfalls noch die Abzeichen mit dem Porträt des damals bereits verstorbenen Reichspräsidenten Friedrich Ebert und dem Emblem für das Reichsbanner Schwarz-Rot-Gold ${ }^{66}$. Der Bedarf der nationalen, konservativen oder vom militärischen Geist geprägten Organisationen an Abzeichen, Orden, Medaillen etc. war allerdings 1926 im Vergleich zu Arbeiterorganisationen und anderen Vereinen und Verbänden erheblich größer. Allein die sogenannten "nationalen Verbände« (Jungdeutscher Orden, Bismarckjugend, Wehrwolf, NSDAP, Deutschvölkischer Jugendbund, Stahlhelm) waren im Katalog mit 51 Artikeln, darunter 15 Abzeichen mit Hakenkreuz-Emblemen, vertreten ${ }^{67}$. Der Bedarf an Abzeichen für Militär-, Krieger-, Kolonial-, Marine-, Kavallerievereine etc., ebenfalls in der Regel der nationalen Rechten zugehörig, war immens, wie der Katalog ausweist ${ }^{68}$. Bei den Parteien hielt die Firma Deumer - Herausgeber dieses Kataloges - für die Deutsch-Nationale-Volkspartei (DNVP), für die Deutsche Volkspartei (DVP) sowie für die Nationalsozialistische Deutsche Arbeiterpartei (NSDAP) Abzeichen vor ${ }^{69}$. Über die eigentlichen Abzeichen hinaus bot die Firma Wilhelm Deumer noch zahlreiche Orden und Ehrenzeichen an - und auch hier begegnet man wieder dem typischen Überhang an Produkten für das konservativ-militärische Spektrum. Nimmt man diesen Überhang als das, was er zu sein scheint, nämlich als das geeignete Angebot für diejenigen, die mit demonstrativem Zurschaustellen ihrer antidemokratischen Gesinnungen die Demokratie aushöhlen wollten, so kann der Firmenkatalog von Deumer als kulturund zugleich organisationsgeschichtliches Zeitdokument ersten Ranges bezeichnet werden ${ }^{70}$.

Die Firmen produzierten im Prinzip für jede politisch-gesellschaftliche Gruppierung. Das wird deutlich an einer der aussagekräftigsten Seiten des Musterbuches der Firma Paulmann \& Crone. Diese Seite führte zahlreiche Abzeichen zum 25jährigen Regierungsjubiläum von Kaiser Wilhelm II. - wie immer uniformiert und von Ordẹn geschmückt - aus dem Jahr 1913 einerseits mit zahlreichen Abzeichen der Sozialdemokratie und Gewerkschaft andererseits zusammen. Auf diesen wurde u.a. der 8 Stunden Tag" und "Gleiches Recht für die Frauen " gefordert - jeweils auf rotem Grund und mit Symbolen wie der aufgehenden Sonne (der sozialistischen Hoffnung) oder den sverschlungenen (Bruder-) Händen (der Solidarität) versehen ${ }^{11}$. Doch auch bei der Firma Paulmann \& Crone gab es bei der Produktion einen deutlichen Überhang national-konservativer und militaristischer Abzeichen. Besonders markante Beispiele hierfür finden sich bei den

65 Ebd., S. 7 f., 10, 21, 37, 39, $63 \mathrm{f}$.

66 Ebd., S. 27.

67 Ebd., S. 26; ebd. auf S. 25 finden sich auch zahlreiche Abbildungen.

68 Ebd., S. 11 f., S. 44-50. Interessant sind die auf den Abzeichen etc. ablesbaren Städtenamen, die belegen, daß Lüdenscheider Firmen wie etwa die Wilhelm Deumer GmbH nahezu überall in Deutschland in jenen Jahren den Bedarf deckten. Kurios ist beispielsweise auch das seinerzeit von Deumer produzierte Abzeichen für den »KriegerVerein Omaruru D.S.W.A.« (Deutsch-Südwestafrika), ebd., S. 49.

69 Ebd., S. 27.

70 Ebd., S. 84-86; eine komplette Aufstellung des Angebotes in diesem Bereich im Jahre 1926 findet sich ebd., S. 87-89.

71 Historisches Firmenarchiv der Firma Paulmann \& Crone GmbH und Co. KG, Musterbuch A1-A2-A3, unpaginiert. 
Objekten, die im Zusammenhang mit dem Ersten Weltkrieg entstanden waren und entsprechend martialische Angriffs- und Vernichtungstöne anschlugen. Auf einem Abzeichen ist eine Granate zu erkennen, deren Kopf von den schwarzweiß-roten Farben und der Aufschrift »Gott strafe England « bestimmt ist. Auf der Granate ist noch ein in die Fluten eintauchendes, kampfbereites U-Boot, am Himmel sind ein Luftschiff und zwei Flugzeuge zu erkennen ${ }^{72}$. Opulent ist später auch das Angebot an Abzeichen für die NSDAP, ihre Unterorganisationen und den nationalsozialistischen Staat ${ }^{73}$, ohne daß eine Datierung der Abzeichen hier exakt möglich wäre. Diese Erzeugnisse gehörten bei vielen Lüdenscheider Firmen - wie wir noch sehen werden - während der Jahre der nationalsozialistischen Herrschaft zu einem vollkommen normalen Produktionsspektrum. Manche Firmen erkannten früher, manche später das darin ruhende Marktpotential.

Der Bedarf konservativer, konservativ-militärischer, militaristischer und nationaler Organisationen, Verbände, Parteien und Vereine - wie auch immer eine Abgrenzung hier zu vollziehen wäre - an dem umrissenen Produktspektrum Lüdenscheider Firmen war grundsätzlich ein höherer als der anderer gesellschaftlicher Bewegungen. Eine ausgeprägte Militarisierung wie bei einer Militärdiktatur, einer straff durchorganisierten faschistischen Diktatur oder einer auf konservativ-militärischen Traditionen fußenden Militärmonarchie wie dem Deutschen Kaiserreich korrespondierte in der Regel mit einer starken gesellschaftlichen Hierarchisierung. Damit war eine dezidierte öffentliche Zurschaustellung gesellschaftlicher Unterschiede an Rang, Einfluß, Verdienst und Reputation verbunden. Dies erhöhte wiederum den Bedarf an medialer Vermittlung. Hierfür stellte die Lüdenscheider Industrie - in dieser Hinsicht führend in Deutschland - die Produkte bereit. Blicken wir unter diesem Gesichtspunkt noch einmal zurück auf den Katalog der Firma Deumer aus dem Jahre 1926, so läßt sich einiges über den Zustand der damaligen Gesellschaft erfahren. Es wurden nun nicht mehr Abzeichen mit Aufschriften wie "Jeder Brit' ein Tritt!« oder "Jeder Stoß ein Franzos'!« produziert, die zur Stärkung des national-kriegerischen Zusammenhaltes während des Ersten Weltkrieges an niederste nationalistische Instinkte appelliert hatten ${ }^{74}$. Der Deumer-Katalog offerierte 1926 vielmehr alle nur erdenklichen Möglichkeiten an differenzierten Vorstandsabzeichen. Über den "Ortsgruppenvorsteher ", "Bundesvorstand ", "Fechtmeister", "Vorturner « bis zum »Turnwartstellvertreter" waren gut 200 (!) Inschriften im Angebot $^{75}$. Abzeichen für Gesangvereine ${ }^{76}$, Radfahrervereine ${ }^{77}$, Schützenvereine $^{78}$, Turnvereine ${ }^{79}$, Sportvereine ${ }^{80}$ sowie Schwimm- und Rudervereine waren im Hinblick auf die Quantität gegenüber den konservativ-militärischen Organisationen zwar, wie erwähnt, unterrepräsentiert, sonst aber untereinànder etwa gleichrangig vertreten, wobei Sänger, Turner und Schützen im Angebot von

Ebd.

Ebd., z.B. Karte Nr. 108.

Ebd.

Deumer, Katalog (wie Anm. 64), S. 4 und S. 28-30.

Ebd., S. 6 f. und S. 32-35.

Ebd., S. 8 und S. 39.

Ebd., S. 9, S. 42 f. und S. 93-97.

Ebd., S. 10 und S. $36 \mathrm{f}$.

Ebd., S. 13 und S. 40. 
Deumer erwartungsgemäß etwas stärker berücksichtigt wurden. Auch Standesbzw. Berufsstandsabzeichen nahmen einen relativ breiten Raum ein. Denn auch Studenten, Bahn- und Postbeamte und eben die große Zahl von Berufen, die in Vereinen, Verbänden und Bruderschaften organisiert waren, hatten alle ihr eigenes Abzeichen und trugen damit dem gesellschaftlichen Leitbild nach Binnendifferenzierung Rechnung ${ }^{81}$.

Viele >nationale Verbände`verfügten in der Weimarer Republik über paramilitärische Einheiten. Zu deren Ausrüstung gehörten im Regelfall auch Koppelschlösser. Trifft die in der Überschrift dieses Beitrages geäußerte Mutmaßung eines engen Zusammenhanges zwischen Militarismus - hier in der Erscheinungsform paramilitärischer Einheiten - und Industrieentwicklung zu, so war auch in diesem Bereich ein breites Angebot der Firmen zu vermuten. Die umfangreichen Offerten der Firma Deumer an Koppelschlössern und Gürtenschnallen bestätigen eine solche Annahme ${ }^{82}$. Diese Entwicklung sollte sich noch eindrucksvoll fortsetzen: Während des >Dritten Reiches< hatten von 93 autorisierten Koppelschloßproduzenten in ,Großdeutschland ' gut 30 ihren Sitz in Lüdenscheid ${ }^{83}$.

\section{Nationalsozialistische Herrschaft}

Alle Lüdenscheider Firmen, die im Deutschen Kaiserreich und in der Weimarer Republik mit der Erzeugung des hier in Rede stehenden Produktsegments befaß̧ waren, setzten ihre Tätigkeit während des >Dritten Reiches` unverändert fort. Firmenleitungen, die sich aus politischen Gründen weigerten, ihre Produkte für den nationalsozialistischen Staat herzustellen, waren für Lüdenscheid nicht zu ermitteln. Ein anderer Befund wäre allerdings auch sehr überraschend gewesen. Rein wirtschaftlich gesehen wirkten sich die Verhältnisse ab 1933 für die Unternehmen zunächst generell sehr positiv aus.

So blickte im Jahr 1939 eine Lüdenscheider Traditionsfirma - sie produzierte u.a. Uniformknöpfe, Militäreffekten und Abzeichen - auf die Entwicklung der letzten Jahrzehnte zurück. Im Rahmen einer Festschrift erzählte sie eine Firmengeschichte - doch diese war typisch für den gesamten kleinteilindustriellen-dekorativ-militaristischen Komplex. Während des Kaiserreichs boten in Deutschland - folgt man dem Tenor der Festschrift - alle Truppengattungen »ein günstiges Absatzgebiet«. »Bei allen Heeresbekleidungsämtern war « dịese Firma »Vertragslieferant. Der große Bedarf des russischen Zarenreichs bot« überdies "ein unbegrenztes Absatzgebiet für hochfeine und veredelte Uniformknöpfe«, das mit dem Ausbruch des Ersten Weltkriegs verlorenging. Das »Kriegsende mit Deutschlands tiefster Erniedrigung und vollkommenen Abrüstung bedeutete für das Geschäft« dieser Firma »den größten Rückschlag, den es in seiner Geschichte erleiden mußte. Der deutsche Binnenmarkt war für Uniformmetalleffekten fast tot.« Zu Beginn der Weimarer Republik schien das Unternehmen dem »Unter-

83 Ray R. Cowdery, Nazi Buckles. Psychology and the Fighting Spirit, Tokyo, Minneapolis, London, Vienna 1982, S. 33-37. 
gang « geweiht, die »Hemmnisse« wirkten "unüberwindlich «. »Da erleichterte der neuauftretende Bedarf an Uniformeffekten für die Gliederungen der NSDAP die Schwierigkeiten. Und als 1933 die große Schicksalswende in der neuesten deutschen Geschichte eintrat, begann auch für die Firma Linden eine Zeit stetigen Aufstiegs. « Der von Hitler 1936 verkündete Vierjahresplan zwang die »Uniformeffektenindustrie« allerdings, sich auf Metalle umzustellen, die in Deutschland abgebaut wurden ${ }^{84}$.

Aus nationalsozialistischer Sicht - und gewiß nicht falsch beurteilt - entsprach die breitflächige Neuverleihung von Orden und Ehrenzeichen durch Staat und Partei weitverbreiteten Wünschen in der Bevölkerung. Der Berliner SA-Sturmführer Cord von Einem, einer der zeitgenössischen Kenner des nationalsozialistischen Auszeichnungswesens, berichtet von den Mentalitäten in der Bevölkerung: "Versetzen wir uns zurück in die Zeit vor dem Kriege [dem Ersten Weltkrieg, E.T.], als die blauen und bunten Uniformen der alten Armee noch das Straßenbild in den Garnisonstädten, insonderheit aber in Berlin, beherrschten. [...] Dieses bunte Bild preußischer Uniformen hat sich in den Jahren während des Krieges und besonders nach dem Kriege vollkommen verwischt. Die Reichswehr bekam neue Abzeichen, die blaue Polizei wurde eingeführt, das allgemeine Interesse schwand, und so wissen wohl heute nur wenige noch mit dem Rangabzeichen und Formationsabzeichen Bescheid." Im Reich Wilhelm II. war dies ganz anders gewesen. "Da wußte jeder, schon die Jungens auf der Schulbank, was er aus bunten Haarbüschen, Tressen und sonstigen Abzeichen zu machen", welchen Regimentern er sie zuzuordnen hatte. "Nach der nationalen Revolution, nachdem nun wieder Uniformen das Straßenbild beherrschen, hat sich das Interesse der Bevölkerung wieder wesentlich gesteigert. Besonders das braune Kleid des S.A.-Mannes belebt das Straßenbild ${ }^{85 .}$ " Die Stimmungslage in der Bevölkerung im Jahre 1934 erscheint hier glaubwürdig wiedergegeben, zumal den Nationalsozialisten die Versöhnung mit den Traditionen des Kaiserreichs bereits am 21. März 1933, am sogenannten Tag von Potsdam, breitenwirksam und langanhaltend erfolgreich gelungen war. Zweifel an solchen Inszenierungen kamen in großen Bevölkerungsteilen erst auf, als der verbrecherische Charakter des Regimes und die Unmöglichkeit eines deutschen Sieges im Zweifrontenkreig offenbar geworden waren. Mit der Wiederbelebung der hypertrophen Uniform- und Abzeichensucht durch die Nationalsozialisten bzw. nach dem Rückfall hinter die demokratische Kultur der Weimarer Republik hatten zahlreiche Firmen die Zeichen der Zeit erkannt und spekulierten auf das bisweilen geradezu erotische Verhältnis zahlreicher ,Volksgenossen` zu Abzeichen, Orden und Kriegsehrungen. Eine zwölfblättrige, in ihren Dimensionen an eine Zeitung erinnernde Werbebroschüre - wahrscheinlich aus dem Jahre 1938 - enthielt in der Anrede an die "geehrte[n] Herren Geschäftsfreunde« das Angebot, auf Wunsch der Kunden die Blätter auf "starken Karton« zu drucken und als "Schaufenster-Reklame« den Lieferungen beizufügen. In kräftigem Rot prangte als Überschrift: »Deut-

Ernst Linden, Gerd Linden und Heinrich Müller, Kurze Geschichte der Knopffabrik Linden, in: Friedrich Linden, Katalog. Uniformeffekten-Fabrik. Knöpfe, Abzeichen, Kokarden, Schulterriemen-Beschläge, Koppelschlösser, Militäreffekten, Lüdenscheid Mai 1939, unpaginiert. Ein Exemplar des Katalogs in Stadtarchiv Lüdenscheid, Sammlung, 180, 241.

85 Abzeichen der SA. Mit Genehmigung der SA-Gruppe Berlin-Brandenburg. Hrsg. von Cord von Einem, Berlin [1934], S. 3. 
scher, trage Deine Kriegs- und Dienstauszeichnungen! « - Verschiedene Ordensdekorationen, Erinnerungsmedaillen, Feldschnallen und Schleifen mit jedem gewünschten Ordensband enthielt dieses Angebot für »Frontkämpfer mit Orden «, »Frontkämpfer «, »Kriegsteilnehmer« und »Kriegshinterbliebene« genauso wie neuere $»$ Dienstauszeichnungen der Wehrmacht ${ }^{86}$.

Kehren wir noch einmal zurück zur bedeutenden Lüdenscheider Abzeichenfirma Wilhelm Deumer. Auch sie legte 1933 und 1939 Kataloge vor. Hochinteressant ist das Druckwerk aus dem Jahr der 'nationalen Revolution`, das allerdings nur Medaillen und Plaketten anbot ${ }^{87}$. Neben dem bei diesem Produktionssegment zu erwartenden Überhang an Produkten für den Sport- und Schützenbereich, die circa 75 Prozent der angebotenen Artikel ausmachten, ist bei diesem Katalog insbesondere das Titelblatt von hohem Aussagewert. Es zeigt auf einer Medaille zusammen Reichspräsident Hindenburg und Reichskanzler Hitler in herzlichem Einvernehmen. Dieses Doppelporträt wurde für Schützenmedaillen, als Abzeichennadel und als großformatige Hitler-Hindenburg-Plakette angeboten. Der zivile Teil der Käuferschicht bei der Firma Deumer entwickelte offenbar einen recht umfangreichen Bedarf an Objekten, durch welche die Botschaft des Tages von Potsdam (21. März 1933), die >Versöhnung des faschistischen HitlerRegimes mit den traditionellen Werten der alten preußischen Militärmonarchie, in sinnfälliger Weise sichtbar gemacht werden konnte ${ }^{88}$.

Im Deumer-Katalog von 1939 sollte diesmal kaum die >Gesellschaft $<$ - wie noch in den Katalogen von 1921, 1926 und 1933 - als Abnehmer angesprochen werden, sondern vor allem Partei und Staat ${ }^{89}$. Fast alle in diesem Druckwerk angebotenen Artikel wurden durch den Staat oder die Partei verliehen. Die Positionen konnten vielfach nur über die Reichszeugmeisterei angefordert werden, die wiederum die Produkte über Ausschreibungen von den Firmen bezog. Der Katalog spiegelte in beeindruckender Weise die außenpolitischen bzw. auch schon militärischen Erfolge des NS-Staates. Neben dem "Spanien-Orden «, der »Österreich-Medaille zur Erinnerung an den 1. Oktober 1938«, der »Protektorats-Spange zur Sudeten-Medaille für den Einmarsch in Böhmen und Mähren« hatte man auch eine »Memel-Medaille zur Erinnerung an den 22. März 1939 « im Programm - der Vormarsch des >Dritten Reiches wird an ihnen deutlich ${ }^{90}$. Der in Lüdenscheid wie in keiner anderen deutschen Stadt angesiedelte Komplex von Firmen, die den Bedarf an Kleinteilen für den dekorativen Militarismus befriedigten, spezialisierte sich zusehends mehr auf Uniformeffekten für die Partei ${ }^{91}$. Die dem Deumer-Katalog beigefügten Blätter geben exakt wieder, an wen Behör-

86 Albert vom Hofe, Lüdenscheid i.W., Katalog: Abzeichen, Orden und Metallwaren, Lüdenscheid [um 1938]. Der Katalog bot neben den bereits erwähnten Produkten zahlreiche ältere Orden und Ehrenzeichen an. Zum Sortiment gehörten auch Emailleabzeichen für Opel und BMW, die bis heute unverändert geblieben sind. Schlüsselanhänger, Einhandvierfarbstifte, Autokleinascher sowie Krawattennadeln und Manschettenknöpfe u.v.a. waren gleichermaßen vertreten.

87 Firma Wilhelm Deumer K.-G., Lüdenscheid, Medaillen und Plaketten. Herbst 1933, [Lüdenscheid] 1933.

88 Ebd., Titelseite, S. 13, 23 und 26.

89 Firma Wilhelm Deumer K.-G., Lüdenscheid, Orden und Ordenartikel. Uniformeffekten. Parteiamtliche Uniform-Effekten, [Lüdenscheid 1933]. Ein Exemplar des Kataloges befindet sich im Stadtmuseum Lüdenscheid.

90 Ebd., S. 4 f.

91 Ebd., S. 46-50. 
denhoheitsabzeichen, Koppelschlösser und Uniformeffekten ausgeliefert werden durften, denn der Erwerb war Privatpersonen in der Regel nicht gestattet. Partei und Staat hatten ein minutiöses System von Berechtigungen entwickelt, mit Hilfe derer dieses Problem geregelt wurde ${ }^{92}$.

Mit der sich abzeichnenden deutschen Niederlage im Zweiten Weltkrieg sollte die hier zum Thema gemachte, spezifisch militärisch-militaristische Tradition zumindest für den westdeutschen Bereich ihr Ende finden. Als am 13. April 1945 amerikanische Truppen vor den Toren Lüdenscheids standen, hoben Privatpersonen, insbesondere aber die verbliebenen Belegschaften einiger Firmen Erdgruben aus, in die hakenkreuzgeschmückte Knöpfe, Orden und Ehrenzeichen kistenweise sentsorgt $\times$ wurden ${ }^{93}$. Damit hatte in dieser Stadt eine besondere Industriekultur zwar nicht ihr vollständiges Ende gefunden, verlor aber in dieser Hinsicht ihr spezifisches Gepräge.

\section{Zusammenfassung}

Werner Sombart beleuchtete 1913 die innere Beziehung von Krieg und Kapitalismus. Er brachte damit einen Zusammenhang in die Diskussion, der vor dem Ersten Weltkrieg fast immer, aber auch nach dem Erscheinen seines Buches häufig vernachlässigt wurde - insbesondere wenn man diese Aussage auf quellenmäßig gut abgestützte Forschungen bezieht. Sombart entwickelte sein Thema an den Verhältnissen des 18. Jahrhunderts mit dem Anliegen, die vielfachen Entstehungsgründe und damit letztlich die Genese des modernen Kapitalismus und der modernen Welt darzustellen. Sombart gewissermaßen als Pionier, aber auch Alexander Gerschenkron und Sidney Pollard sowie viele andere haben sich mit den unterschiedlichen Dimensionen des Industrialisierungsprozesses, dessen regionalen Besonderheiten sowie zeitlichen Verzögerungs- und Beschleunigungsfaktoren beschäftigt ${ }^{94}$. Es besteht heute in der Forschung Konsens darüber, daß in der Zeit zwischen 1850 und 1873 der Produktionskapitalismus seinen "großen Spurt « erlebte und daß der inneren Entwicklung dieses Kapitalismus dabei die "verstärkten Impulse seines >Wachstumskerns` zugute« kamen, der seit den vierziger Jahren des 19. Jahrhunderts auch für die Zeitgenossen deutlich erkennbar wurde. Der Eisenbahnbau, die Eisen- und Stahlproduktion und der Bergund Maschinenbau waren dessen Leitsektoren ${ }^{95}$. Der vorliegende Beitrag ging von der in der Geschichts- und Wirtschaftwissenschaft allgemein akzeptierten These aus, daß der Militärbedarf an ‘schwerem Gerät ‘ eine sehr förderliche Wirkung auf die Entwicklung des Produktionskapitalismus ausübte, bald sogar zur in: Industrielle Revolution. Wirtschaftliche Aspekte. Hrsg. von Rudolf Braun [u.a.], Köln, Berlin 1972, S. 59-82, und Sidney Pollard, Peaceful Conquest. The Industrialisation of Europe 1760-1970, Oxford 1981. Zur Rolle der Region im Industrialisierungsprozeß vgl. dort bes. S. 111-123.

95 Wehler, Deutsche Gesellschaftsgeschichte (wie Anm. 53), S. 67.

Eingefügt ebd. zwischen S. 46 und 47.

Mündliche Überlieferung einiger Lüdenscheider Familien, insbesondere von Firmeninhabern.

Alexander Gerschenkron, Wirtschaftliche Rückständigkeit in historischer Perspektive, 
Ausbildung eines militärisch-industriellen Komplexes führte. Innerhalb der märkisch-bergischen Wirtschaftsregion, deren 'großer Spurt، nicht so rasant verlief wie im benachbarten Ruhrgebiet, partizipierten - wie wir am Beispiel Lüdenscheids zeigten - zahlreiche Industriebetriebe mit Metallkleinteilprodukten ebenfalls an den immensen Möglichkeiten, die mit der Ausbildung gigantischer Armeen im In- und Ausland einhergingen. Diese Betriebe erzeugten mit Blick auf die deutschen Verhältnisse für eine sich zusehends urbanisierende, bevölkerungsmäßig nahezu potenzierende, industrialisierende und militarisierende Gesellschaft. Der Bedarf dieser Gesellschaft nach industriell gefertigten Metallkleinteilen wurde noch dadurch erheblich gesteigert, daß sich in ihr ein hypertropher Sozialmilitarismus ausbreitete, der breitflächig. und nachhaltig nach dekorativem Ausdruck verlangte. Die in Lüdenscheid wie in keiner anderen deutschen Stadt sich vollziehende, vielfach von der gesamtgesellschaftlichen Militarisierung bedingte und überlagerte Sonderform der Industrialisierung ist zwar in ihrer Art einzigartig gewesen, doch ähnliche Entwicklungen in abgeschwächter Form haben sich auch in anderen Städten des bergisch-märkischen Wirtschaftsraums vollzogen. Deren Erforschung anzuregen und damit einen Beitrag zur Erhellung der Geschichte der deutschen Industrialisierung und dessen Produktionskapitalismus zu leisten, war eine der Absichten dieses Beitrages. Zweitens war intendiert, einen bislang weitgehend unbeachteten Aspekt des deutschen Militarismus aufzuhellen. Wehler spricht davon, daß im Deutschen Kaiserreich die Uniform von Soldaten und Offizieren sogar im Urlaub mit »pfauenhaftem Stolz« getragen wurde. "Auch nach dem aktiven Dienst boten Aufmärsche und nationale Feste, Kaisergeburtstagsfeiern und private Vergnügungen immer wieder einen Anlaß«, sich in seiner Uniform zu präsentieren. Diese zutreffende Feststellung bezieht sich zwar auf die militärische Uniform, doch sie beansprucht Gültigkeit in einem viel weiteren Sinn, denn durch die Bismarcksche Reichseinigung und die sie voraussetzenden militärischen Erfolge waren Uniformen insgesamt, Orden, Abzeichen und Ehrenzeichen auf dem »Gipfel der Prestigehierarchie«, zumindest aber extrem populär und verbreitet ${ }^{96}$. Ein dritter Aspekt erscheint bedeutsam: In diesem Beitrag sind Originalobjekte beschrieben und mit Abbildungen versehene Firmenkataloge analysiert worden. Dies könnte im Hinblick auf die Überlieferung von Originalobjekten in der als historisches Zentrum für die Produktion von Kleinteilen des dekorativen Militarismus beschriebenen Stadt Lüdenscheid noch wesentlich vertieft werden. Diese Objekte sind kulturelle Objektivationen eines hypertrophen Militarismus und machen diesen durch ihre spezifische Aura geradezu sinnlich erfahrbar. Unser Aufsatz versteht sich daher auch als Versuch, eine an gesellschaftsgeschichtlichen Fragestellungen orientierte Militärhistorie mit spezifisch kulturhistorischen Fragestellungen zu verknüpfen.

Diese Untersuchung hat in einem circa 300 Jahre umspannenden Überblick versucht, den durch den Armeebedarf und den Befürfnissen sozial-militarisierter Organisationen ausgelösten Boom spezifischer, häufig uniformschmückender Metallkurzwaren - soweit auf kurzem Raum möglich - in einen politik-, gesellschafts- und industriegeschichtlichen Gesamtzusammenhang einzuordnen und diesen Prozeß anhand der Stadt Lüdenscheid aufzugreifen, die sich wegen ihrer spezifischen Industriestruktur wie kaum eine andere zur Darstellung die- 
ses Zusammenhanges als geeignet erwies. Ohne in wünschbarer Komplexität einen umfassenden Nachweis führen zu können, ist doch deutlich geworden, daß sich Lüdenscheid als Sonderfall der deutschen Industrialisierung darstellt. Die Stadt nämlich entwickelte sich u.a. über die Befriedigung von Militärbedarf und die Ausrüstung der vielfach konservativ-militärischen Organisationen und Verbände mit Abzeichen und Plaketten von einer im Jahre 1800 handwerklich geprägten Stadt mit circa 1500 Einwohnern zu einer Metropole deutscher und weltweiter Bedarfsbefriedigung im oben genannten Sinne. Lüdenscheid konnte 1907 dann auch schon 31115 Einwohner nachweisen. Selbst wenn man die Entwicklung der Stadt insbesondere im 19. Jahrhundert gewiß nicht auf diesen militärischen Aspekt reduzieren darf, denn Andenken, Bestecke, Modeartikel u.a.m. wurden ebenfalls produziert, so machen doch - wie oben geschildert die Produktsegmentspezialisierung und -intensivierung in den siebziger und achtziger Jahren des 19. Jahrhunderts und später die Entwicklung von einigen Lüdenscheider Traditionsfirmen hin zu fast ausschließlichen Zulieferbetrieben für Partei, Armee und Staat im >Dritten Reich < deutlich, daß die spezifische Bedarfsentwicklung von sich stets stärker militarisierenden, uniformierenden und hierarchisierenden Gesellschaften einen prägenden Einfluß auf regionale Industrieentwicklungen ausüben konnte. 